\title{
Indolo[2,3-a]quinolizidines and derivatives: Bioactivity and asymmetric synthesis
}

\author{
Maria Pérez, ${ }^{a}$ Margarida Espadinha, ${ }^{b}$ Maria M. M. Santos ${ }^{\mathrm{b}, *}$
}

\begin{abstract}
a Laboratory of Organic Chemistry, Faculty of Pharmacy and Institute of Biomedicine (IBUB), University of Barcelona, Av. Joan XXIII, s/n, 08028 Barcelona, Spain

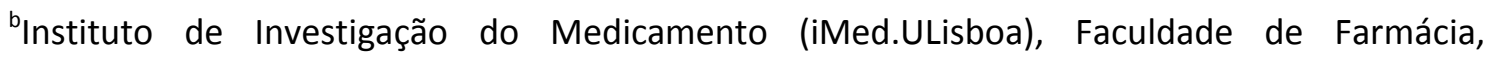
Universidade de Lisboa, Av. Prof. Gama Pinto, 1649-003 Lisboa, Portugal
\end{abstract}

\section{Corresponding Author}

* Maria M. M. Santos, Instituto de Investigação do Medicamento (iMed.ULisboa), Faculdade de Farmácia, Universidade de Lisboa

Av. Prof. Gama Pinto, 1649-003 Lisboa, Portugal, Tel:+351 217946451, Fax: +351 217946470, mariasantos@ff.ulisboa.pt

RUNNING TITLE: Indoloquinolizidines and derivatives 


\begin{abstract}
Corynantheine alkaloids with a tetracyclic indole[2,3-a]-quinolizidine motif are an important issue in academia and in the life science industries due to their broad bioactivity profile ranging from antiarthritic, analgesic, anti-inflammatory, antiallergic, antibacterial, to antiviral activities. For that reason, in the last decades, numerous efforts have been invested in the development of novel synthetic strategies to obtain the indole[2,3-a]-quinolizidine system. This review focuses on the synthetic methodologies developed to target the most important alkaloids of this family, and highlights the potential use of these alkaloids or analogs to treat several diseases, ranging from cancer to neurodegenerative disorders.
\end{abstract}

Keywords: Indoloquinolizidines; Corynantheine alkaloids; Indole alkaloids; Natural products; Bioactivity 


\section{Introduction}

Corynantheine alkaloids with a tetracyclic indole[2,3-a]-quinolizidine motif are of great interest for academia and pharmaceutical companies due to their broad bioactivity profile. In fact, this type of indole alkaloids have been described with different biological activities, ranging from antiarthritic, analgesic, anti-inflammatory, to anti-cancer activities. For this reason, in the last decades, numerous efforts have been invested in the development of novel synthetic strategies to obtain the indolo[2,3-a]quinolizidine system present in numerous monoterpenoid-derived alkaloids.[1-3] Since the chirality of the corynantheine alkaloids is crucial for the expression of bioactivity, there is a huge interest in the development of new enantioselective synthesis to obtain indolo[2,3-a]quinolizidine alkaloids. In particular, several research studies (development of novel synthetic strategies and biological evaluation) have been conducted with corynantheine alkaloids, such as dihydrocorynantheine (1), corynantheine (2), dihydrocorynantheol (3), hirsutine (4), hirsuteine (5), and geissoschizine methyl ether (6) (Figure 1). This review focus on the pharmacological and therapeutic interest of indolo[2,3-a]quinolizidines and the most recent advances in asymmetric synthetic strategies to obtain the main skeleton of these indole alkaloids. All the papers described in this review were found using Scifinder, PubMed, and Web of Science databases.
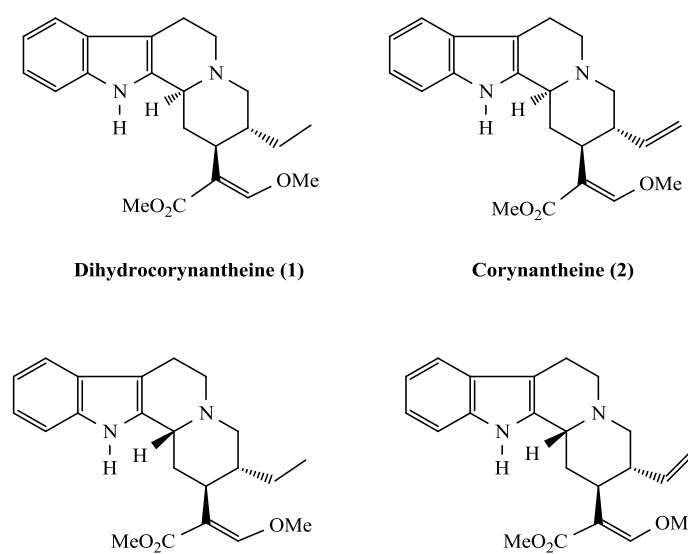

Hirsutine (4)
Corynantheine (2)

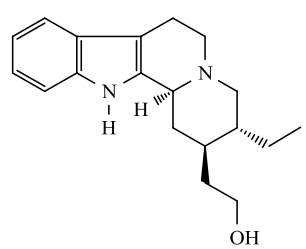

Dihydrocorynantheol (3)

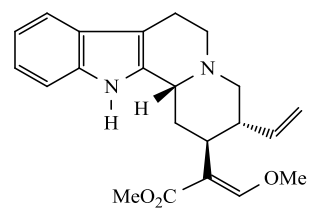

Hirsuteine (5)

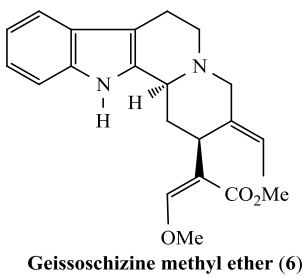

Figure 1 - Examples of corynantheine alkaloids. 


\section{Biological activities of indolo[2,3-a]quinolizidine alkaloids}

\subsection{Arborescidine A and desbromoarborescidine A}

In 1966 , it was described the isolation of 1,2,3,4,6,7-hexahydro- $12 H$-indolo[2,3-a]quinolizine alkaloid (latter called desbromoarborescidine A) from Dracontomelum mangiferum.[4] The cardiovascular activities of desbromoarborescidine A (7) and five natural product analogs 8-12 (Figure 2) were evaluated by studying the effect of these alkaloids in blocking Alpha-1 and Alpha-2 adrenoceptors. Desbromoarborescidine A (7) showed a strong blocking activity of the adrenoceptors compared with the other 5 alkaloids. In fact, the presence of a hydroxyl group at position 1 led to loss of activity.[5]

In 1993, it was described the isolation of the brominated indole alkaloid arborescidine A (13) from the marine tunicate Pseudodistoma arborescens.[6] The antiproliferative activity of desbromoarborescidine A (7) and arborescidine A (13) (Figure 2) was evaluated in vitro against several tumor cell lines (lung fibroblasts, gastric adenocarcinoma, leukemia and others). Arborescidine A (13) showed an $\mathrm{IC}_{50}$ value of $34.5 \mu \mathrm{M}$ for leukemia, while desbromoarborescidine A (7) was weakly active in all tumor cell lines tested $\left(\mathrm{IC}_{50}\right.$ higher than $50 \mu \mathrm{M}) \cdot[7]$

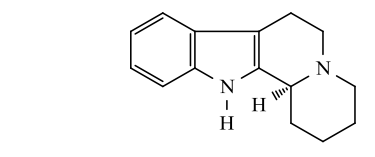

Desbromoarborescidine A (7)

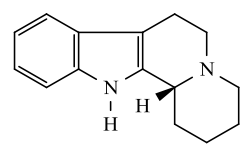

8

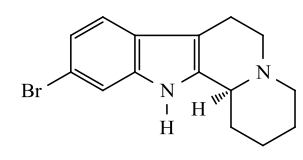

Arborescidine A (13)

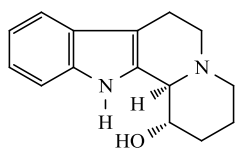

9

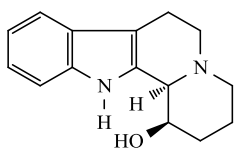

10

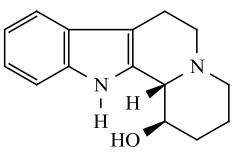

11

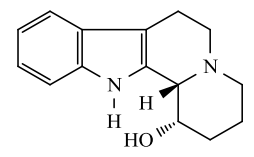

12

Figure 2 - Chemical structure of desbromoarborescidine A (7), indoloquinolizidines 8-12, and arborescidine A (13). 


\subsection{Dihydrocorynantheine (1), corynantheine (2), and derivatives}

In the late 80 's Chang et al. reported the effects of dihydrocorynantheine (1) (see Figure 1) in arterial pressure. The alkaloid was extracted from the dried leaves and stems of Uncaria Callophylla and the studies demonstrated that dihydrocorynantheine (1) can effectively lower the arterial pressure in both anaesthetized and conscious normotensive rats.[8]

Later, Masumiya et al. described that dihydrocorynantheine (1) showed direct effects on the potential action of cardiac muscle through inhibition of multiple ion channels, which could explain their negative chronotropic and antiarrhythmic activity.[9]

In 2000, dihydrocorynantheine (1), corynantheine (2), and corynantheidine (14) were isolated from bark of Corynanthe pachyceras K. Schum. (Rubiaceae). These alkaloids, and two corynantheidine racemic synthetic derivatives 15-16 (Figure 3), were tested against Leishmania major promastigotes. All compounds were active for Leishmania, presenting $\mathrm{IC}_{50}$ values between 0.7 and $2.8 \mu \mathrm{M}$. The three alkaloids were also evaluated for antiplasmodial and cytotoxic activities but revealed to be inactive.[10]

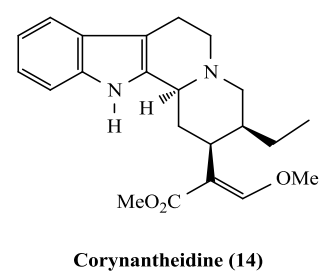

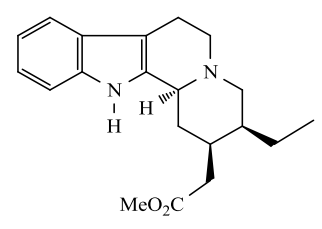

15

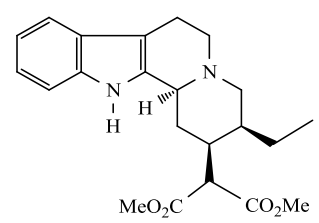

16

Figure 3 - Chemical structure of corynantheidine (14) and two synthetic derivatives 15-16.

\subsection{Dihydrocorynantheol (3) and derivatives}

In 2011, Fröde's research group reported for the first time the anti-inflammatory effects of dihydrocorynantheol (3) (see Figure 1), an alkaloid isolated from Esenbeckia leiocarpa. Dihydrocorynantheol (DHC) (3) was shown to play a pivotal role in the anti-inflammatory effect exercised by this herb by preventing the I $\kappa \mathrm{B} \alpha$ ubiquitination and consequent degradation, inhibiting thus the NF- $\mathrm{KB}$ cascade and, consequently, the production of several proinflammatory mediators, such as IL-1 $\beta$ and TNF- $\alpha .[11]$ 
A series of ester dihydrocorynantheol derivatives (DHC-acetyl 17, DHC-p-methylbenzoyl 18, DHC-benzoyl 19, DHC-p-methoxybenzoyl 20 and DHC-p-chlorobenzoyl 21) (Figure 4) were also tested as anti-inflammatory agents. It was observed that protection of the hydroxyl group resulted in a decrease of activity, which indicates that the presence of a hydroxyl group in the chemical structure of dihydrocorynantheol (3) is important for the activity of this alkaloid against inflammation.[12]

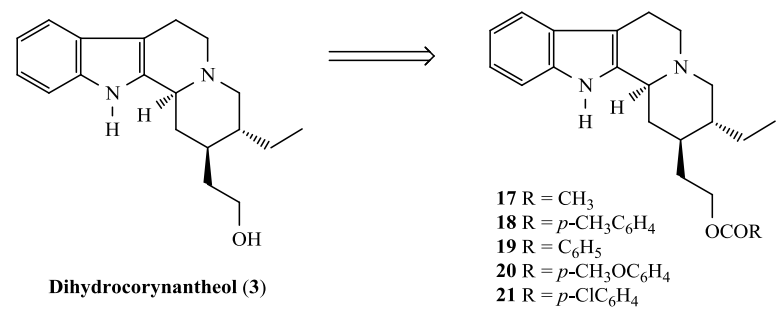

Figure 4 - Chemical structure of dihydrocorynantheol (3) and indoloquinolizidines 17-21.

\subsection{Hirsutine (4), hirsuteine (5), geissoschizine methyl ether (6) and derivatives}

Hirsutine (4), hirsuteine (5) and geissoschizine methyl ether (6) (see Figure 1) are the primary constituents of Uncaria sp. Hirsutine (4), isolated from Uncaria rhynchophylla (traditional Chinese herb medicine), was described to possess antihypertensive and antiarrhythmic activities through modulation of the intracellular $\mathrm{Ca}^{2+}$ levels in rat thoracic aorta[13] and action potential in cardiac muscle[9]. Moreover, hirsutine (4) was shown to be effective in the protection of rat cardiomyocytes from hypoxia-induced cell death.[14] Moreover, the effects of hirsutine (4), hirsuteine (5) and geissoschizine methyl ether (6) (Figure 1), extracted from Uncariae Ramulus et Uncus, were evaluated on vascular responses. Geissoschizine methyl ether (6) proved to be 14 times more active $\left(\mathrm{EC}_{50}=0.744 \mu \mathrm{M}\right)$ than hirsutine $(\mathbf{4})$, in norepinephrine-induced vasocontractive response. Also, geissoschizine methyl ether (6) was shown to have two different mechanisms of action: endothelium dependency with nitric oxide and endothelium independency with voltage-dependent $\mathrm{Ca}^{2+}$ - channel blocking. Therefore, geissoschizine methyl ether (6) might be a candidate for vasodilative or antihypertensive medicines.[15]

In 2011, villocarine A (Figure 5) was isolated from the leaves of Uncaria villosa (Rubiaceae). In that report, the authors describe villocarine $\mathrm{A}$ as a new indole alkaloid, however the structure 
is identical to the indole alkaloid 3-epi-geissoschizine methyl ether (22). Villocarine A showed potent vasorelaxant effects at $30 \mu \mathrm{M}$ in rat aortic ring assays, revealed some inhibition effect on vasocontraction of depolarized aorta with high concentration potassium, and showed inhibition effect on phenylephrine (PE)-induced contraction in the presence of nicardipine in a $\mathrm{Ca}^{2+}$ concentration-dependent manner.[16, 17]

In 2011, T. Ueda et al. demonstrated that geissoschizine methyl ether (6) is a partial agonist at the serotonin 5- $\mathrm{HT}_{1 \mathrm{~A}}$ receptor, a partial agonist/antagonist at the dopamine $\mathrm{D}_{2 \mathrm{~L}}$ receptor and an antagonist at the serotonin $5-\mathrm{HT}_{2 \mathrm{~A}}, 5-\mathrm{HT}_{2 \mathrm{C}}$ and $5-\mathrm{HT}_{7}$ receptors. The pharmacological profiles of geissoschizine methyl ether (6) were similar to aripiprazole (commercial drug used for the treatment of schizophrenia and related disorders), however, geissoschizine methyl ether (6) was less potent than aripiprazole at dopamine $\mathrm{D}_{2 \mathrm{~L}}$ receptors $\left(\mathrm{EC}_{50}=4.4 \mu \mathrm{M}\right.$ for geissoschizine methyl ether (6) vs. $\mathrm{EC}_{50}=56 \mathrm{nM}$ for aripiprazole).[18] Moreover, in 2012, gissoschizine methyl ether (6) was described as a potential acetylcholinesterase inhibitor. In this study, hirsutine (4), hirsuteine (5), and vallesiachotamine (23) (Figure 5), extracted from the hooks of Uncaria Rhynchophylla, were also tested. The results showed an inhibition of $50 \%$ of acetylcholinesterase activity at concentrations of $3.7 \pm 0.3 \mu \mathrm{g} \mathrm{mL}^{-1}$ for geissoschizine methyl ether (6). Hirsutine (4), hirsuteine (5), and vallesiachotamine (23) were weakly active against acetylcholinesterase.[19] Moreover, turbinatine (24) (Figure 5),[20] a corynanthean-type indole alkaloid, with a similar structure with geissoschizine methyl ether (6), inhibited acetylcholinesterase with an $\mathrm{IC}_{50}$ of $0.99 \mu \mathrm{g} \mathrm{mL}^{-1}$.[19]

The efficacy of hirsutine (4) on neuroinflammation control was also explored. It was shown that hirsutine (4) reduces the production of several neurotoxic factors in activated microglial cells and possesses neuroprotective activity in a model of inflammation-induced neurotoxicity. In particular, in organotypic hippocampal slice cultures, hirsutine (4) blocked lipopolysacchariderelated hippocampal cell death and production of nitric oxide, prostaglandin $\mathrm{E}_{2}$ and interleukin$1 \beta .[21]$

In 2014, a new alkaloid, 4-geissoschizine $N$-oxide methyl (25) (Figure 5), was isolated from the hook-bearing branch of Uncaria rhynchophylla. The neuroprotective effects of this compound, 
and 5 other alkaloids [hirsutine (4), hirsuteine (5), 4-hirsuteine $\mathrm{N}$-oxide (26), geissoschizine methyl ether (6), 3-epi-geissoschizine methyl ether (22)] were evaluated against 3mM glutamate-induced HT22 cell death. The 4-geissoschizine $N$-oxide methyl ether (25) and 4hirsuteine $\mathrm{N}$-oxide (26) compounds revealed to be weak neuroprotective agents. On the other hand, the remaining compounds showed potent neuroprotective effects against glutamate induced HT22 cell death.[22]

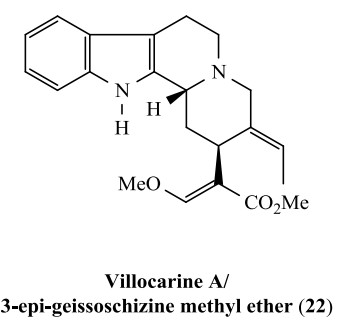

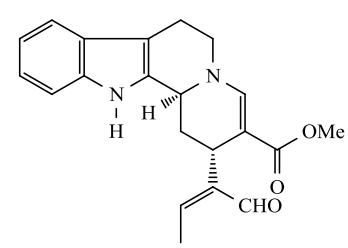

Vallesiachotamine (23)

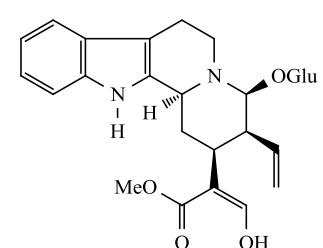

Turbinatine (24)
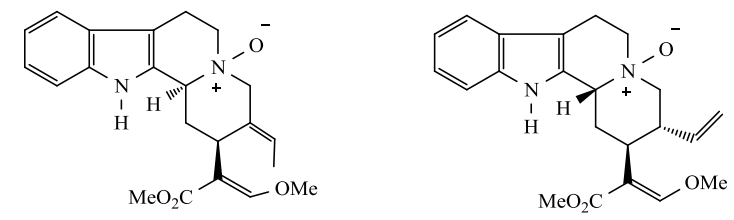

4-Geissoschizine $N$-oxide methyl ether (25)

4-Hirsuteine $N$-oxide (26)

Figure 5 - Chemical structure of 3-epi-geissoschizine methyl ether (22), vallesiachotamine (23), turbinatine (24), 4-geissoschizine $N$-oxide methyl ether (25), 4-hirsuteine $N$-oxide (26).

Hirsutine (4) was also identified as an anti-metastatic by targeting nuclear factor-kB activation from a screening of 56 natural product derivatives. In particular, hirsutine (4) strongly suppressed NF-kB activity in murine $4 \mathrm{~T} 1$ breast cancer cells, reducing the metastatic potential of $4 \mathrm{~T} 1$ cells. Moreover, hirsutine (4) reduced the in vivo lung metastatic potential of $4 \mathrm{~T} 1$ cells. These results indicate that hirsutine (4) can be an attractive lead compound for reducing the metastasis potential of cancer cells.[23]

\subsection{Arboricinine and arboricine}


Arboricinine (27) and arboricine (28) (Figure 6) were isolated from stem-bark extract of the Malayan Kopsia arborea. These indoloquinolizidines revealed moderate cytotoxicity against $\mathrm{KB} / \mathrm{VJ} 300$ cell lines, a vincristine-resistant human oral epidermoid carcinoma, with $\mathrm{IC}_{50}$ values around $30 \mu \mathrm{M} .[24]$

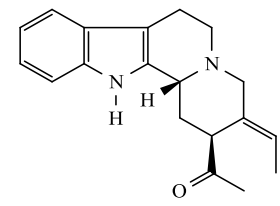

Arboricinine (27)

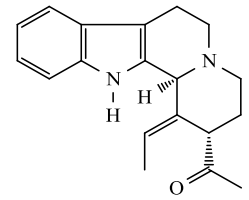

Arboricine (28)

Figure 6 - Chemical structure of arboricinine (27) and arboricine (28).

\subsection{Vallesiachotamine}

In 1981, Z-vallesiachotamine (29) (Figure 7), a monoterpene indole alkaloid, isolated from Rhaza stricta, was reported to have anticancer properties on a carcinoma cell line.[25] Latter, the in vitro antiproliferative activity of vallesiachotamine isolated from the leaves of Policourea rigida was investigated on human melanoma cells. The compound presented an $\mathrm{IC}_{50}$ value of 14.7 $\mu \mathrm{M}$ in SK-MEL-37 melanoma cells (two times more active than doxorubicin), induced accumulation of melanoma cells in the G0/G1 growth phase and increased the proportion of sub-G1 hypodiploid cells (at $11 \mu \mathrm{M}$ and $22 \mu \mathrm{M}$ ). Moreover, at $50 \mu \mathrm{M}$, vallesiachotamine caused extensive cytotoxicity and necrosis.[26]

Vallesiachotamine was also tested for therapeutic targets involved in neurodegeneration. In particular, extracts obtained from Psychotria laciniata containing Z-vallesiachotamine (29), and E-vallesiachotamine (30) (Figure 7) as major compounds, showed high potency against monoamine oxidase A (MAO-A) and only moderate potency against monoamine oxidase B (MAO-B).[27] Moreover, Z-vallesiachotamine (29), E-vallesiachotamine (30), and vallesiachotamine lactone (31) (Figure 7) were shown to inhibit butyrylcholinesterase (BChE) and $\mathrm{MAO}-\mathrm{A}$ with $\mathrm{IC}_{50}$ values ranging from 7.08 to $14 \mu \mathrm{M}$ for $\mathrm{BChE}$ inhibition and from 0.85 to $2.14 \mu \mathrm{M}$ for MAO-A inhibition.[28] Finally, using a computational structure-based approach, it was investigated if these three alkaloids bind sirtuin 1 and sirtuin 2 . The compounds 
demonstrated a SIRT1 inhibitory profile comparable to that of sirtinol (nonspecific SIRT inhibitor). Opposite to $Z$-vallesiachotamine (29) and E-vallesiachotamine (30), vallesiachotamine lactone (31) demonstrated no apparent toxicity on Hek 293 and on rat astrocyte primary cells.[29] These findings are in line with the study of Z-vallesiachotamine (29) on human melanoma cells.[26]
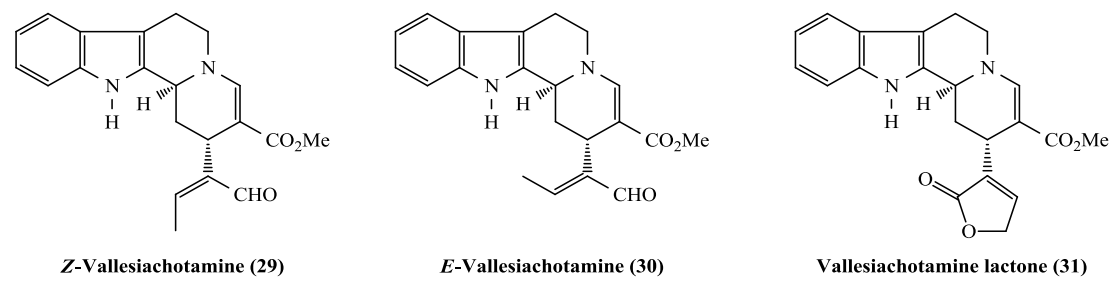

Figure 7 - Chemical structure of $Z$-vallesiachotamine (29), $E$-vallesiachotamine (30), and vallesiachotamine lactone (31).

\subsection{Mitragynine and derivatives}

In 2005, Matsumoto et al. have studied the effect of mitragynine (32) (Figure 8), a major indolealkaloid founded in Thai medicinal herb Mitragyna speciosa, on neurogenic contraction of smooth muscle. The results demonstrated that mitragynine (32) inhibited the contraction of guinea-pig vas deferens produced by electrical transmural stimulation. More precisely, mitragynine (32) was found to block T- and L-type $\mathrm{Ca}^{2+}$ channel currents and reduced $\mathrm{KCl}$ induced $\mathrm{Ca}^{2+}$ influx in N1E-115 neuroblastoma cells.[30]

One year later, 7-hydroxyspeciociliatine (33) (Figure 8) was isolated, for the first time, from the fruits of Malaysian Mitragyna speciosa Korth. The opioid agonistic activity of this alkaloid and 7-hydroxymitragynine (34) was investigated in guinea-pig ileum experiments. The results demonstrated that 7-hydroxyspeciociliatine (33) had a weak stimulatory effect on $\mu$-opioid receptors,[31] while 7-hydroxymitragynine (34) had moderate opioid agonist activity, as reported previously.[32, 33]

Also, 9-demethyl analogue of mitragynine, 9-hydroxycorynantheidine (35) (Figure 8), synthesized from mitragynine, was reported as a partial agonist of opioid receptors. The 
receptor binding assays revealed that 9-hydroxycorynantheidine (35) has affinity for three opioid receptor types. In particular, 9-hydroxycorynantheidine (35) (Figure 8) presented $\mathrm{pK}_{\mathrm{i}}$ values of 7.92, 4.51 and 5.53 , for $\mu-, \delta$ - and $\kappa$-opioid receptors, respectively. The results show that 9-hydroxycorynantheidine (35) has a high affinity and selectivity for $\mu$-opioid receptors.[34]

Also, recently Shamima et al. investigated the action of mitragynine (32) as antinociceptive agent. The goal of this study was to understand if mitragynine (32) acts on Cannabinoid receptor type 1. The results demonstrated that mitragynine (32) doesn't act on Cannabinoid receptor type 1 but through activation of opioid receptor system, more precisely on $\mu$ - and $\delta$ opioid receptors.[35]

More recently, two derivatives of 7-hydroxymitragynine (compounds $\mathbf{3 6}$ and 37, Figure 8), were synthesized in order to develop dual-acting $\mu$ - and $\delta$-opioid agonists. Compound $\mathbf{3 7}$ was shown to be more potent, in vitro and in vivo, than compound $\mathbf{3 6}$ and 7-hydroxymitragynine (34). Compound 37 exhibited a high affinity for $\mu$ - and $\delta$-opioid receptors, with $\mathrm{K}_{\mathrm{i}}$ values of 2.1 and $7.0 \mathrm{nM}$, respectively, while compound 36 reveled $\mathrm{K}_{\mathrm{i}}$ values of $6.4 \mathrm{nM}$ ( $\mu$-opioid receptor) and $16.0 \mathrm{nM}$ ( $\delta$-opioid receptor). Moreover, the antinociceptive effect of compound $\mathbf{3 7}$ was approximately 240 times more potent than that of morphine in a mouse tail-flick test and, for this reason compound $\mathbf{3 7}$ could be used as potential therapeutic agent for treating neuropathic pain.[36] 


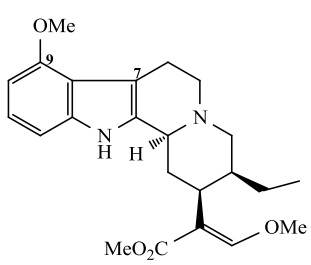

Mitragynine (32)

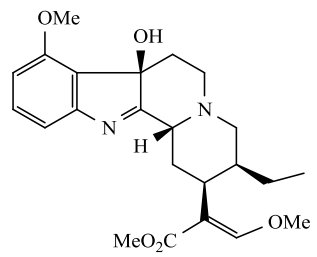

7-Hydroxyspeciociliatine (33)

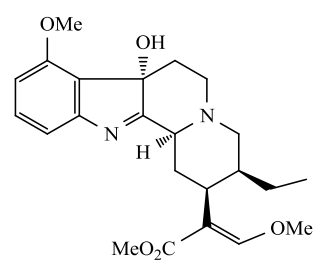

7-Hydroxymitragynine (34)

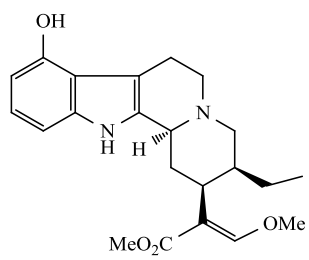

9-Hydroxycorynantheidine (35)

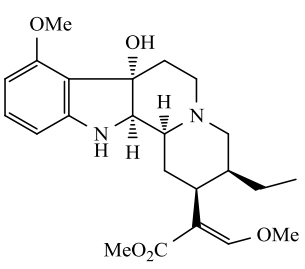

36

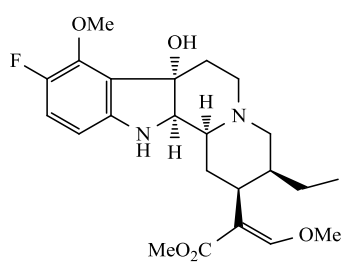

37

Figure 8 -Chemical structure of mitragynine (32), 7-hydroxyspeciociliatine (33), 7-

hydroxymitragynine (34), 9-hydroxycorynantheidine (35) and indoloquinolizidines 36-37.

\subsection{Non-natural indolo[2,3-a]quinolizidine derivatives}

Takanawa et al., reported the synthesis of 10 mitragynine derivatives in order to perform a structure-activity relationship study. The rational was based on the fact that mitragynine (32) has analgesic activity. The effects of corynantheidine (14) (Figure 3), mitragynine (32) (Figure 8), synthetic derivatives 35, 38-46, and speciociliatine (47) (Figure 9) were evaluated on opioid receptors, using electrically stimulated contraction in isolated guinea pig ileum. Seven compounds (compounds 32, 35, 42-43, and 45-47) revealed an interesting potency against opioid receptors (Graph 1).

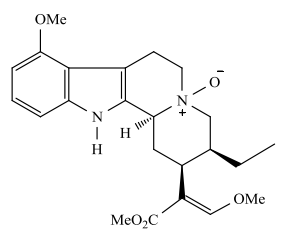

38

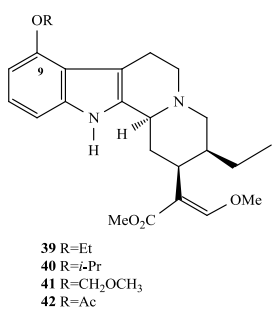

$39 \mathrm{R}=\mathrm{Et}$
$40 \mathrm{R}=i-\mathrm{Pr}$
$41 \mathrm{R}=\mathrm{CH}$
$42 \mathrm{R}=\mathrm{Ac}$

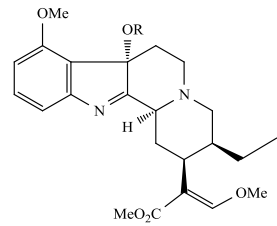

$43 \mathrm{R}=\mathrm{Ac}$
$44 \mathrm{R}=\mathrm{H}$

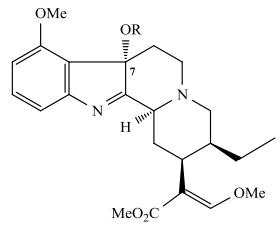

$45 \mathrm{R}=\mathrm{Me}$
$46 \mathrm{R}=\mathrm{Et}$

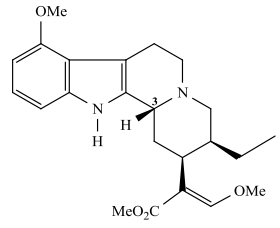

Speciociliatine (47)

Figure 9 - Chemical structure of mitragynine derivatives 38-46 and speciociliatine (47). 
The potency of mitragynine (32) revealed to be one-fourth of morphine (standard compound that has a relative potency of $100 \%$ ). The 9-demethoxy analogue of mitragynine, i.e. corynantheidine (14), did not show any opioid agonistic activity. However, analysis of its effects on twitch contraction inhibited by morphine, atropine, and verapamil in electrically stimulated guinea pig ileum, showed that corynantheidine (14) inhibits the effect of morphine via functional antagonism of opioid receptors. On the other hand, 9-hydroxycorynantheidine (35) inhibited electrically induced twitch contraction in guinea pig ileum and revealed relative potency higher than mitragynine (32). The introduction of the acetoxy group on the indole ring (compound 42), led to marked reduction in both intrinsic activity and potency as compared with those of mitragynine (32) (Graph 1). Mitragynine derivative 41 did not show any opioid agonist activity, which indicates that the functional groups at the C9 position are very important for the activity of these compounds.

Speciociliatine (47), a C-3 stereoisomer of mitragynine (32), could be found as a minor constituent on Mitragyna speciosa. This compound reveled to be 14 -fold weaker than mitragynine (32). The introduction of a methoxy or an ethoxy group at the C-7 position, respectively, compounds $\mathbf{4 5}$ and $\mathbf{4 6}$, led to a dramatic reduction in potency for opioid receptors and, which indicates that the hydrogen atom at the C-7 position in mitragynine (32) has an important role for the activity. Finally, compound 44, showed a relative potency 13 - and 46-fold higher for opioid receptors than those of derivative 39 and mitragynine (32), respectively. Compound $\mathbf{4 4}$ demonstrated affinity also for $\delta$ - and $\kappa$-receptors.

The relative affinities of compounds $\mathbf{1 4}, \mathbf{3 2}, 35$ and $\mathbf{4 4}$, for the three opioid receptors $(\mu, \delta$ and $\kappa)$, were determined using receptor binding assay (Graph 2) using morphine as comparison standard. The results show that these compounds have relatively high selectivity for $\mu$-receptors. Moreover, mitragynine (32) revealed some affinity for $\delta$-receptor, while compound $\mathbf{4 4}$ demonstrated affinity for both $\delta$ - and $\kappa$-receptors.[37] 


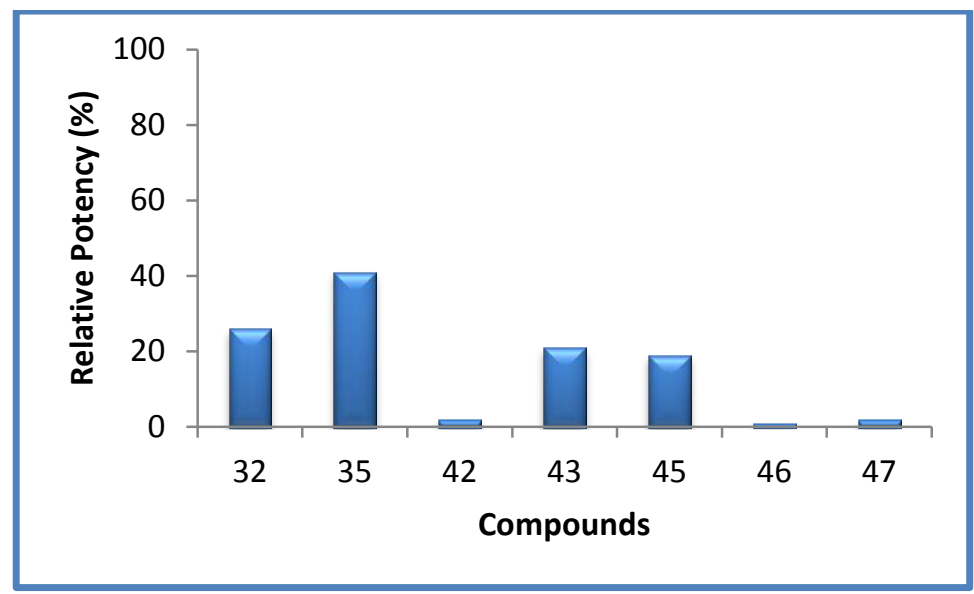

Graph 1 - Relative potency of seven compounds with interesting activities for opioid receptor.

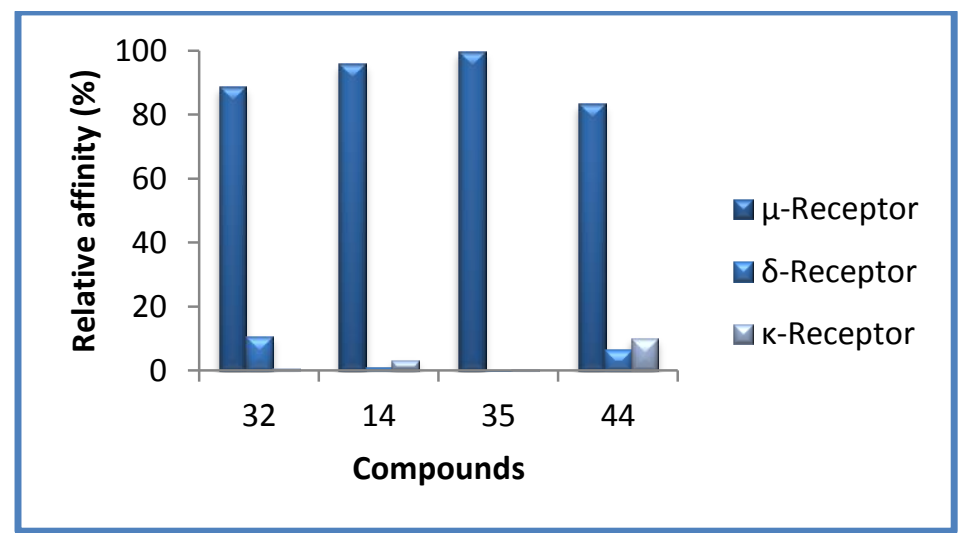

Graph 2 - Relative affinity of indoloquinolizidines $14,32,35,44$ for $\mu, \delta$, and $\kappa$ receptor types.

Waldmann et al. evaluated a collection of approximately 11000 natural-product derived and inspired compounds as apoptosis inducers. From that library, seven indoloquinolizidine derivatives 48-54 (Figure 10) were considered potential apoptosis inducers in three human tumor cell lines, presenting values of $\mathrm{IC}_{50}$ around $2 \mu \mathrm{mol} \mathrm{L}{ }^{-1}$ in HeLa (cervix), MCF-7 (breast) cell lines and in HepG2 (liver, with slightly lower efficiency) cell lines.[38] 


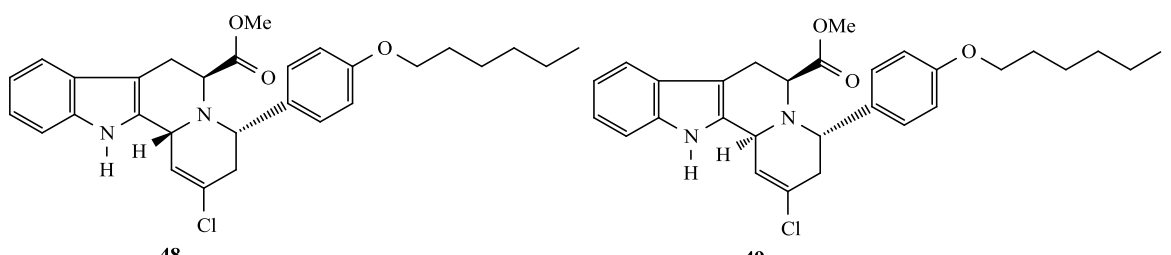

48

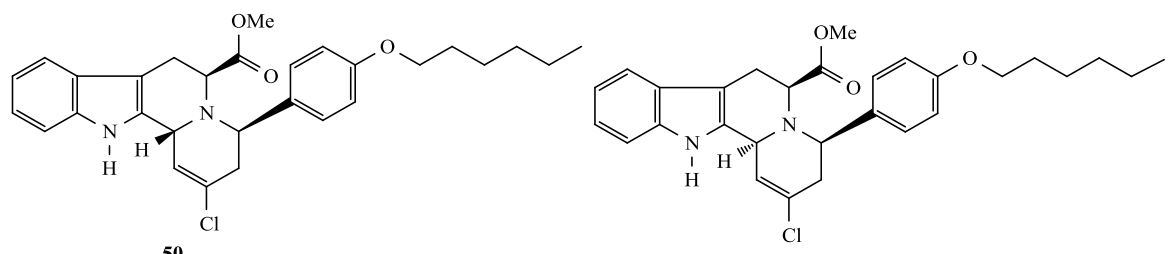

50

51

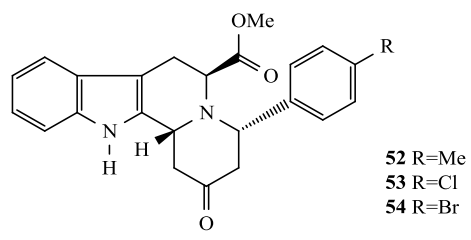

Figure 10 - Chemical structure of indoloquinolizidine derivatives 48-54.

A series of 20 indolo[2,3- $a$ ]quinolizidine-peptide hybrids was synthesized and evaluated for $\mathrm{D}_{1}$ dopamine receptors $\left(D_{1} R\right)$ and $D_{2}$ dopamine receptors $\left(D_{2} R\right)$. Two diastereomeric indolo[2,3a]quinolizidines were coupled with tripeptides in order to enhance the affinity of the indoloquinolizidine moiety for the dopamine receptors. Several compounds presented higher affinities than dopamine. Furthermore, it was shown that trans $(\mathrm{C}-3$ and $\mathrm{C}-12 \mathrm{~b})$ indoloquinolizidine derivatives had stronger effect in the interaction with the receptors than cis indoloquinolizidine derivatives (Figure 11).[39] The functional characterization of the hybrid compound $\mathbf{6 1}$ by means of kinetic assays and competition experiments in radioligand binding, demonstrated that indoloquinolizidine-peptide $\mathbf{6 1}$ behaves as an orthosteric ligand of dopamine $D_{2}, D_{3}, D_{4}$ and $D_{5}$ receptors, but as a negative allosteric modulator of agonist and antagonist binding to striatal dopamine $D_{1}$ receptors. In addition, compound $\mathbf{6 1}$ decreased receptor potency, while preserving agonist-induced maximal cAMP production.[40] 

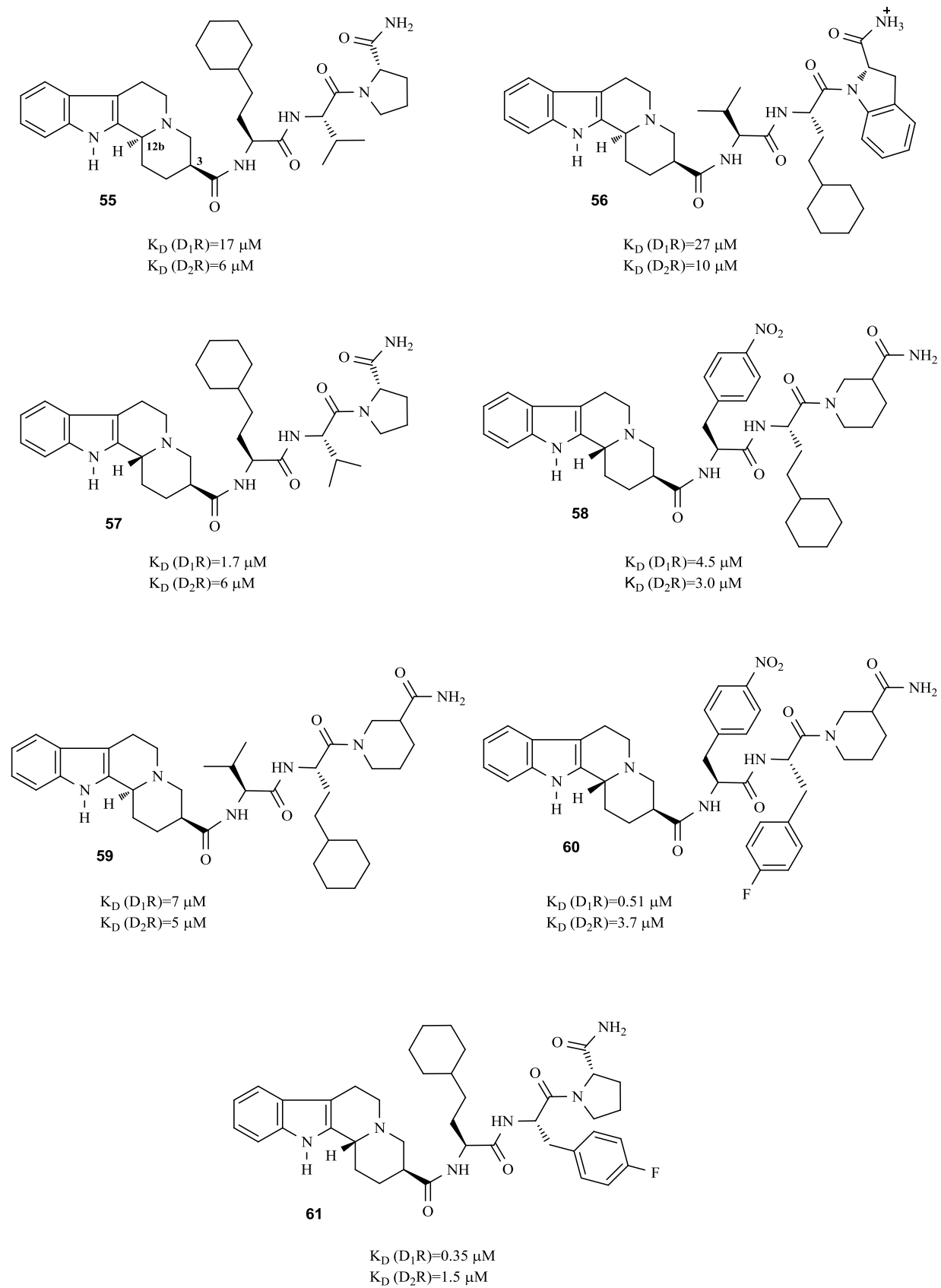

Figure 11 - Most active indoloquinolizidine-peptide hybrids.

In 2015, a novel indoloquinolizidine derivative 62 (Figure 12) was synthesized and evaluated as an anti-hypertension agent. The compound showed remarkable antihypertensive and dilating effect both in vitro and in vivo. Moreover, it was shown that compound $\mathbf{6 2}$ induced vasodilatation by both endothelium-dependent and-independent manners, blocked $\mathrm{Ca}^{2+}$ influx through L-type $\mathrm{Ca}^{2+}$ channels and inhibited intracellular $\mathrm{Ca}^{2+}$ release while not affecting $\mathrm{K}^{+}$ channel.[41] 


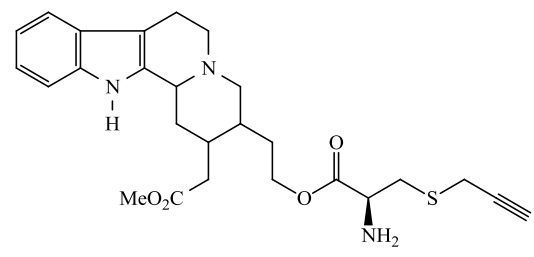

62

Figure 12 - Chemical structure of indoloquinolizidine derivative $\mathbf{6 2}$.

In tables 1 and 2 the reader can find a summary of the main biological properties described to date for Indolo[2,3-a]quinolizidines and derivatives. 
Table 1 - Biological properties of Indolo[2,3-a]quinolizidines and derivatives.

\begin{tabular}{|l|l|l|l|}
\hline & Species isolated from & & \multicolumn{1}{|c|}{ Effects } \\
\hline
\end{tabular}




\begin{tabular}{|c|c|c|c|}
\hline Hirsutine (4) & Uncaria rhynchophylla & $\begin{array}{l}\text { - antihypertensive and antiarrhythmic activities } \\
\text { - reduces the production of several neurotoxic factors in activated microglial cells and } \\
\text { possesses neuroprotective activity in a model of inflammation-induced neurotoxicity } \\
\text { - potent neuroprotective effects against glutamate induced HT22 cell death } \\
\text { anti-metastatic }\end{array}$ & $\begin{array}{l}{\left[\begin{array}{ll}9, & 13-14, \\
21-23\end{array}\right]}\end{array}$ \\
\hline & & - potent neuroprotective effects against glutamate induced HT22 cell death & [22] \\
\hline $\begin{array}{c}\text { OMe } \\
\text { Geissoschizine methyl ther (6) }\end{array}$ & $\begin{array}{l}\text { Uncariae Ramulus et } \\
\text { Uncus }\end{array}$ & $\begin{array}{l}\text { - Active in norepinephrine-induced vasocontractive response } \\
\text { partial agonist at the serotonin 5-HT1A receptor, a partial agonist/antagonist at the } \\
\text { dopamine D2L receptor and an antagonist at the serotonin 5-HT2A, 5-HT2C and 5- } \\
\text { HT7 receptors } \\
\text { - potential acetylcholinesterase inhibitor } \\
\text { - potent neuroprotective effects against glutamate induced HT22 cell death }\end{array}$ & $\begin{array}{ll}15, & 18-19, \\
22] & \end{array}$ \\
\hline
\end{tabular}




\begin{tabular}{|l|l|l|}
\hline & - active for Leishmania \\
\hline Pangiferum & - blocking of Alpha-1 and Alpha-2 adrenoceptors \\
\hline
\end{tabular}




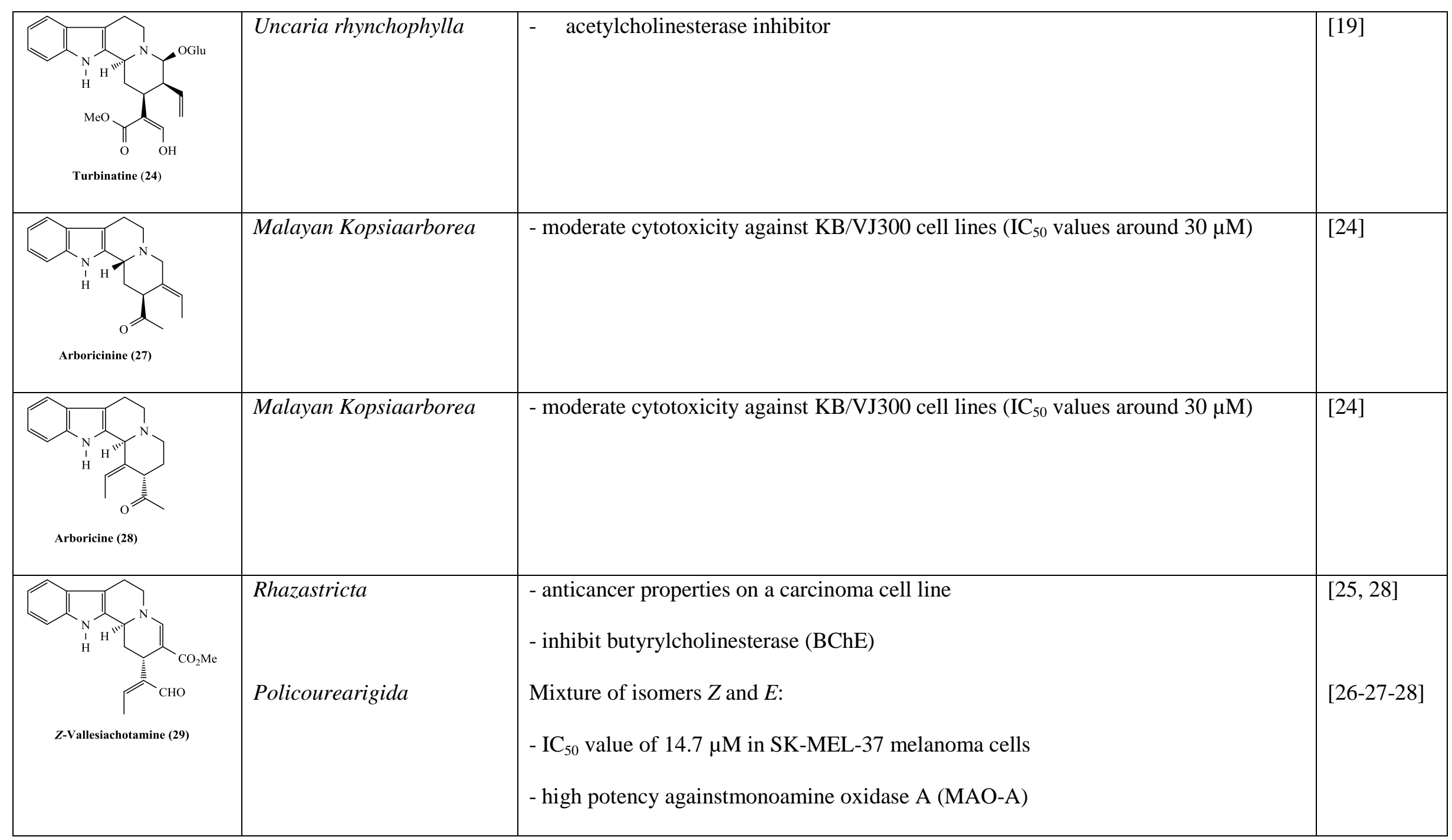




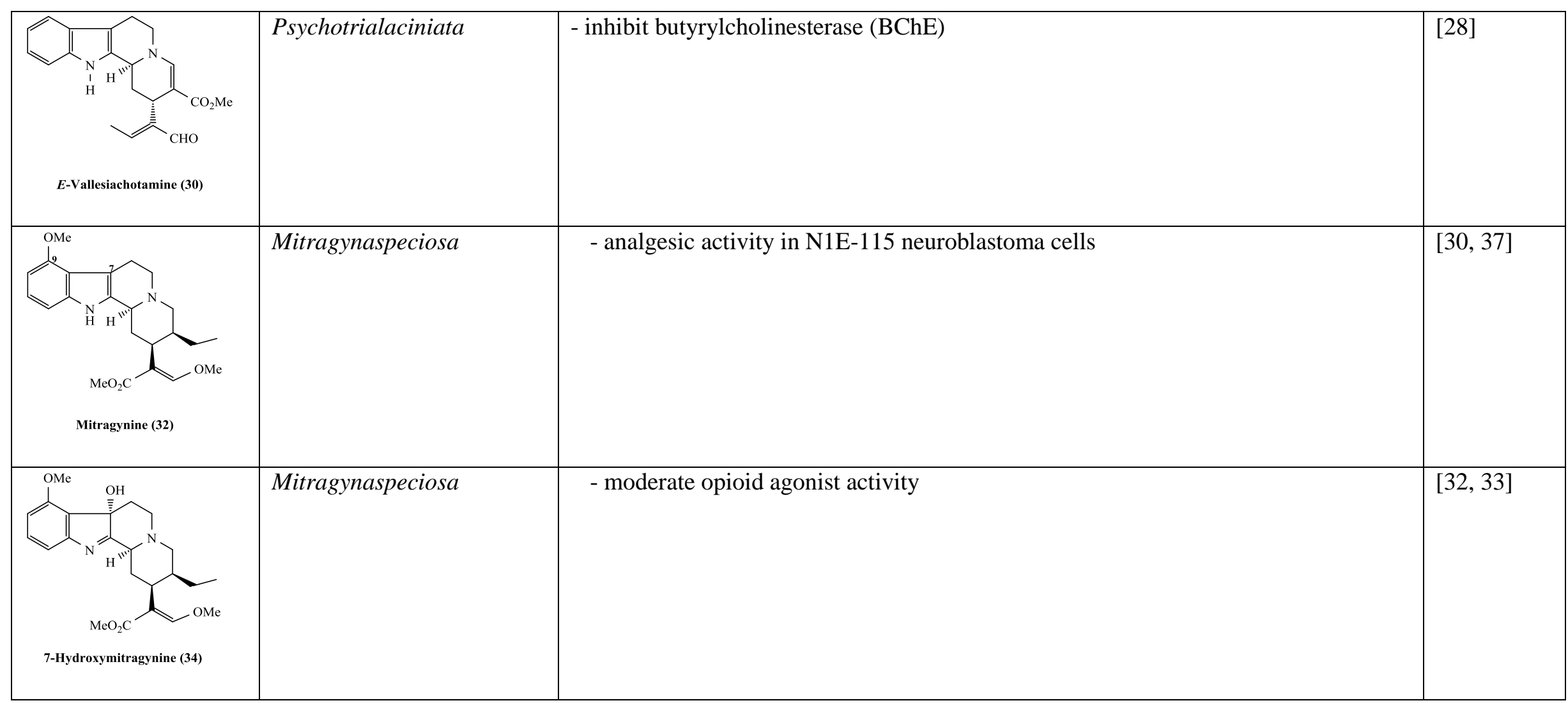


Table 2 - Biological properties of Indolo[2,3-a]quinolizidine derivatives.

\begin{tabular}{|l|l|l|}
\hline & Effects \\
\hline - partial agonist of opioid receptors, high affinity and selectivity for $\mu$-opioid receptors
\end{tabular}




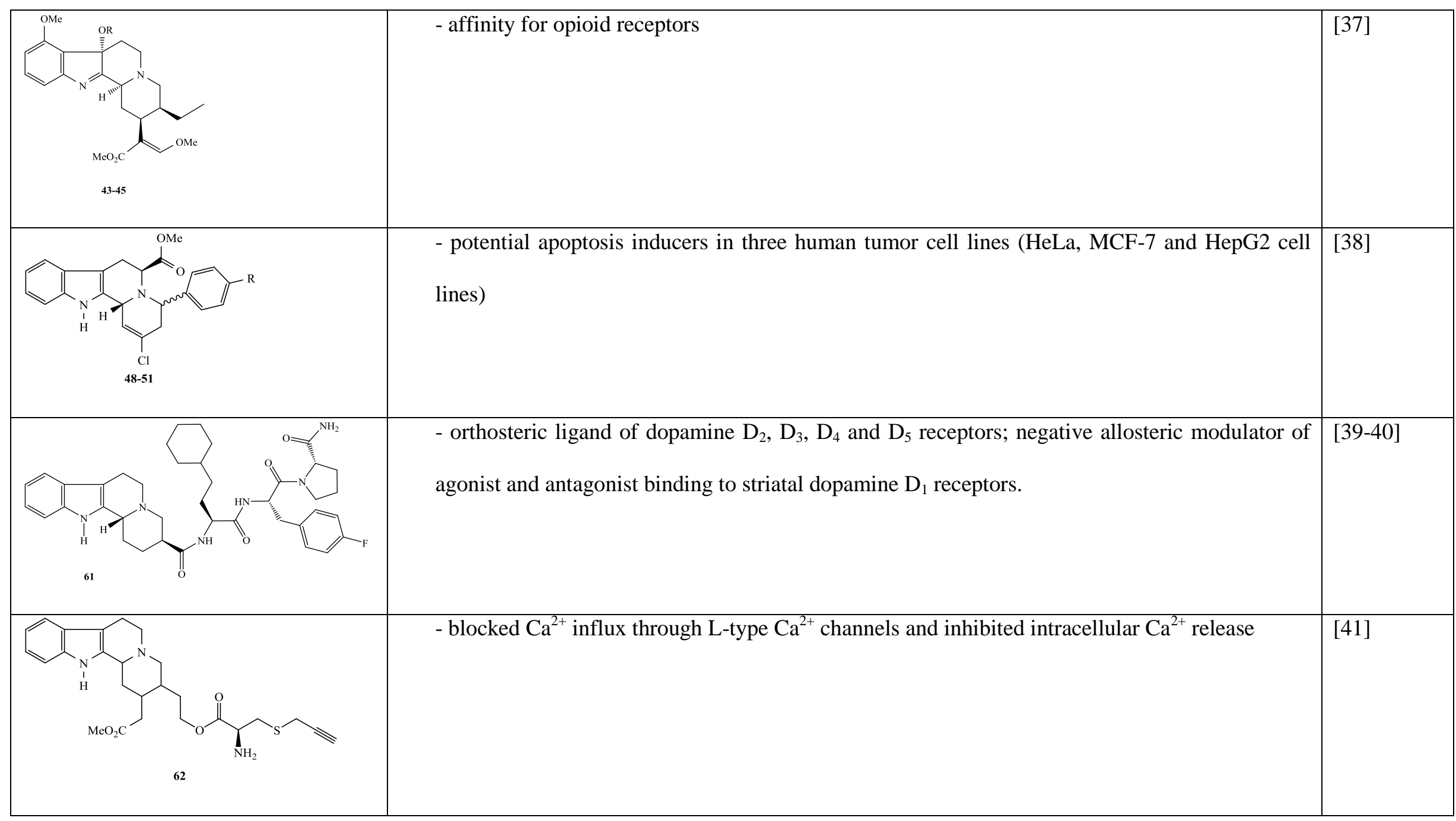




\section{Synthesis of indolo[2,3-a]quinolizidine alkaloids}

The aim of this part of the review is to present the state of the art of the different strategies developed in enantioselective indolo[2,3-a]quinolizidine alkaloid synthesis, illustrated with representative examples. It is divided in three sections, namely, use of chiral pool resources, non-catalytic cascade/tandem sequences; and asymmetric metalcatalysis/organocatalysis approaches. From a synthetic point of view, construction of the fused-ring system of the indolo[2,3-a]quinolizidine alkaloids with control of the relative and absolute stereochemistry of the quinolizidine core represents a significant challenge that makes these natural products attractive synthetic targets. Traditionally, the majority of reported strategies for the asymmetric total synthesis of indolo[2,3-a]quinolizidine alkaloids have required a multistep synthesis that relies on starting materials from the chiral pool. These strategies often include several functional group transformations and tedious protection/deprotection steps, often providing a low overall yield of the target alkaloid. In the last decades cascade reactions have been applied in indoloquinolozidine alkaloids synthesis. Although extremely demanding, the well stablished advantages of these reactions include atom economy, as well as economies of time, labor, resources management, and waste generation. In this section we have include a significant racemic synthesis. Most recently there have been a few reports of efficient syntheses of optically active indoloquinolizidine natural products based on asymmetric catalysis. This approach is highly efficient and productive. However, the majority of the developed synthetic strategies is target-specific with respect to the relative configuration of the quinolizidine stereocenters and only allows for selective formation of one specific epimer of the alkaloid.

\subsection{Use of chiral pool resources}

Chiral pool synthesis makes use of chiral natural products by incorporating part of them into the target structure. As either enantiomer of the desired final product can be potentially generated, compounds from the chiral pool are extremely valuable and versatile in asymmetric synthesis. For many decades, it was the only source of enantiomerically pure compounds, but nowadays 
many of the most effective chiral agents described in the literature have been designed and synthesized by organic chemists.

\subsubsection{Lactim ether route: Enantioselective synthesis of (-)-dihydrocorynantheol (3)}

Prof. Tozo Fujii has published several papers on fused quinolizidine ring systems, including the total synthesis of (-)-dihydrocorynantheine (1) in 1991.[42] The "lactim ether route" requires the coupling of the 3-(chloroacetyl)indole with the lactim ether $\mathbf{6 5}$, prepared from cincholoipon ethyl ester (64, obtainable from commercially available (+)-cinchonine $(\mathbf{6 3})$ by the classical degradation procedure[43]). Subsequent treatment of the resulting keto derivative $\mathbf{6 6}$ with $\mathrm{POCl}_{3}$ afforded the corresponding oxazolium chloride, which was then reduced by catalytic hydrogenation to furnish the lactam 67 . The conversion into the tetracyclic ester $\mathbf{6 8}$ was carried out in $91 \%$ overall yield by means of Bischler-Napieralski cyclization followed by catalytic hydrogenation. Final $\mathrm{LiAlH}_{4}$-reduction of $\mathbf{6 8}$ afforded (-)-dihydrocorynantheol (3) in quantitative yield (Scheme 1).
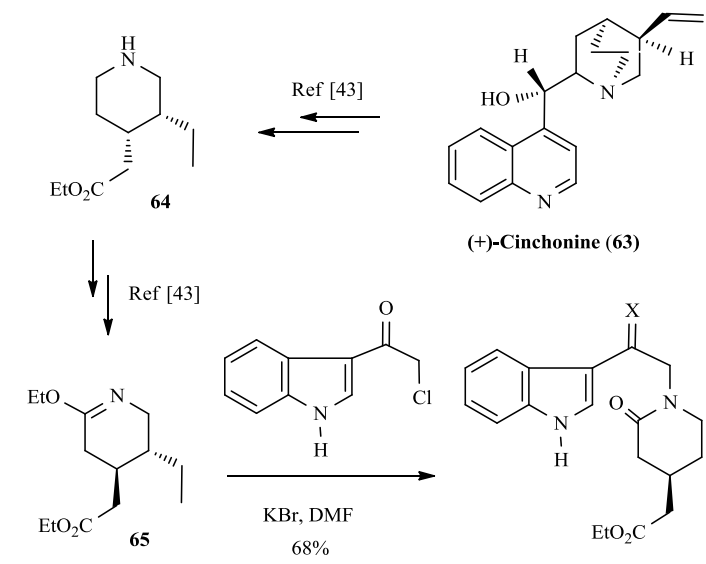

(+)-Cinchonine (63)
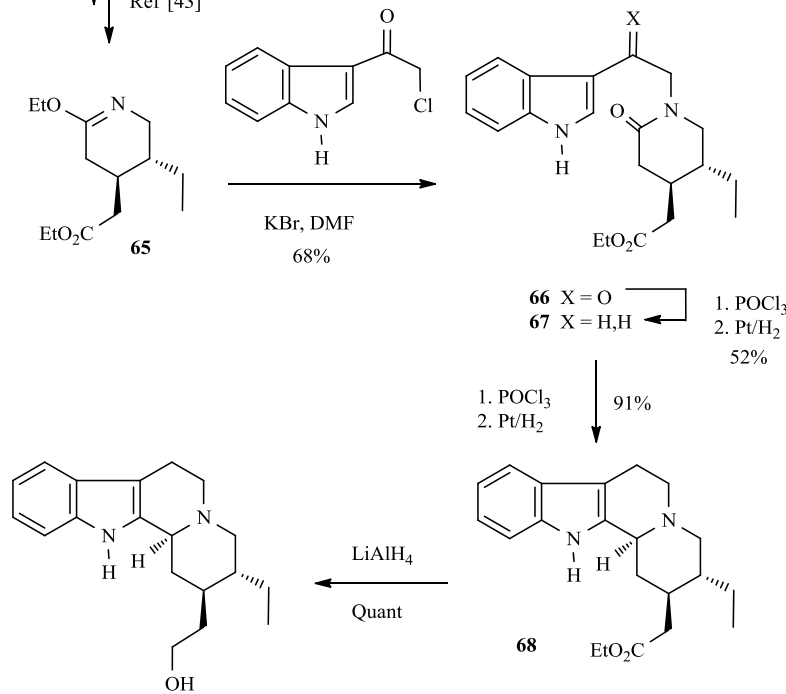

(-)-Dihydrocorynantheol (3)

Scheme 1 - Enantioselective synthesis of (-)-dihydrocorynantheol (3). 
The usefulness of the "lactim ether route" in the asymmetric synthesis of Corynanthe-type indoloquinolizidine alkaloids is demonstrated by the application of this methodology to the synthesis of (-)-ochromianine (73), (-)-ophiorrhizine (74), and (-)-ochropposinine (75), employing the lactim ether $\mathbf{6 5}$ as starting material (Scheme 2).
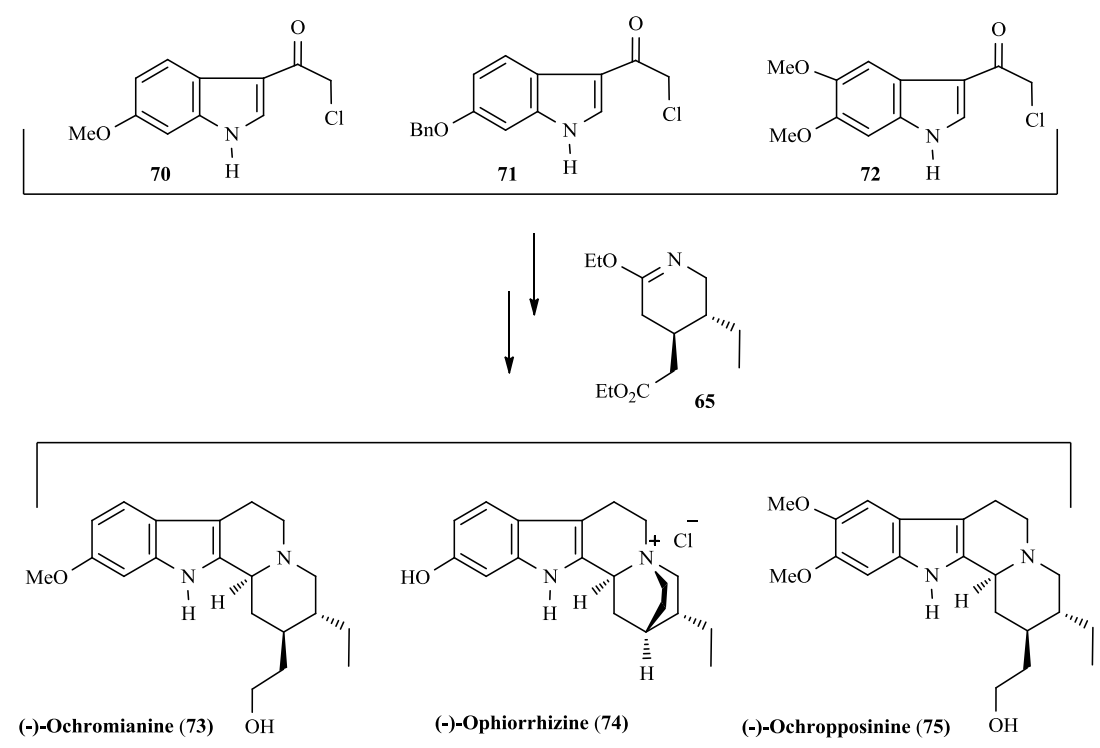

Scheme 2 - Enantioselective synthesis of of (-)-ochromianine (73), (-)-ophiorrhizine (74), and (-)-ochropposinine (75).

\subsubsection{Stereoselective Mannich reaction from a $(R)$-tryptophan derivative: Enantioselective} synthesis of (+)-geissoschizine (76)

A concise synthesis of (+)-geissoschizine (76) (Scheme 3), a biosynthetic precursor of a variety of monoterpenoid indole alkaloids,[1] from $(R)$-tryptophan was performed by Stephen F. Martin in 2003.[44]

The synthesis started with a vinylogous Mannich reaction involving the iminium ion 78, which was prepared from $(R)$-tryptophan (77). The corresponding adduct was then treated directly with isobutylene in the presence of sulfuric acid to give $\mathbf{8 0}$ as the only isolable product. The nucleophilic attack of the vinyl ketene $\mathbf{7 9}$ on $\mathbf{7 8}$ occurred with high diastereoselectivity from the si face, establishing the correct absolute stereochemistry at C-3 of geissoschizine. $N_{\mathrm{b}}$-Acylation 
of $\mathbf{8 0}$ with diketene furnished an intermediate $\beta$-keto amide that underwent facile cyclization via an intramolecular Michael reaction upon addition of potassium tert-butoxide to give 81. This reaction, which presumably proceeded under thermodynamic control to establish the correct relative stereochemistry at $\mathrm{C}-3$ and $\mathrm{C}-5$, completed the assembly of the corynantheane framework.

Toward the introduction of the $E$-ethylidene side chain, hydride reduction of the C-19 carbonyl function in $\mathbf{8 1}$, followed by a stereoselective dehydration, led to the stereoselective elimination to give the ester 82. The subsequent selective reduction of the lactam function according to the Borch protocol[45] furnished $\mathbf{8 3}$ in $92 \%$ yield. Cleavage of the tert-butyl ester moiety was achieved using trifluoroacetic acid in the presence of thioanisole, as an essential cation scavenger. The cleavage of the carboxyl group at C-5 was accomplished by a radical decarbonylation of an acyl selenide intermediate. A final formylation by Winterfeldt's procedure[46] afforded (+)-geissoschizine (76) in $48 \%$ yield.

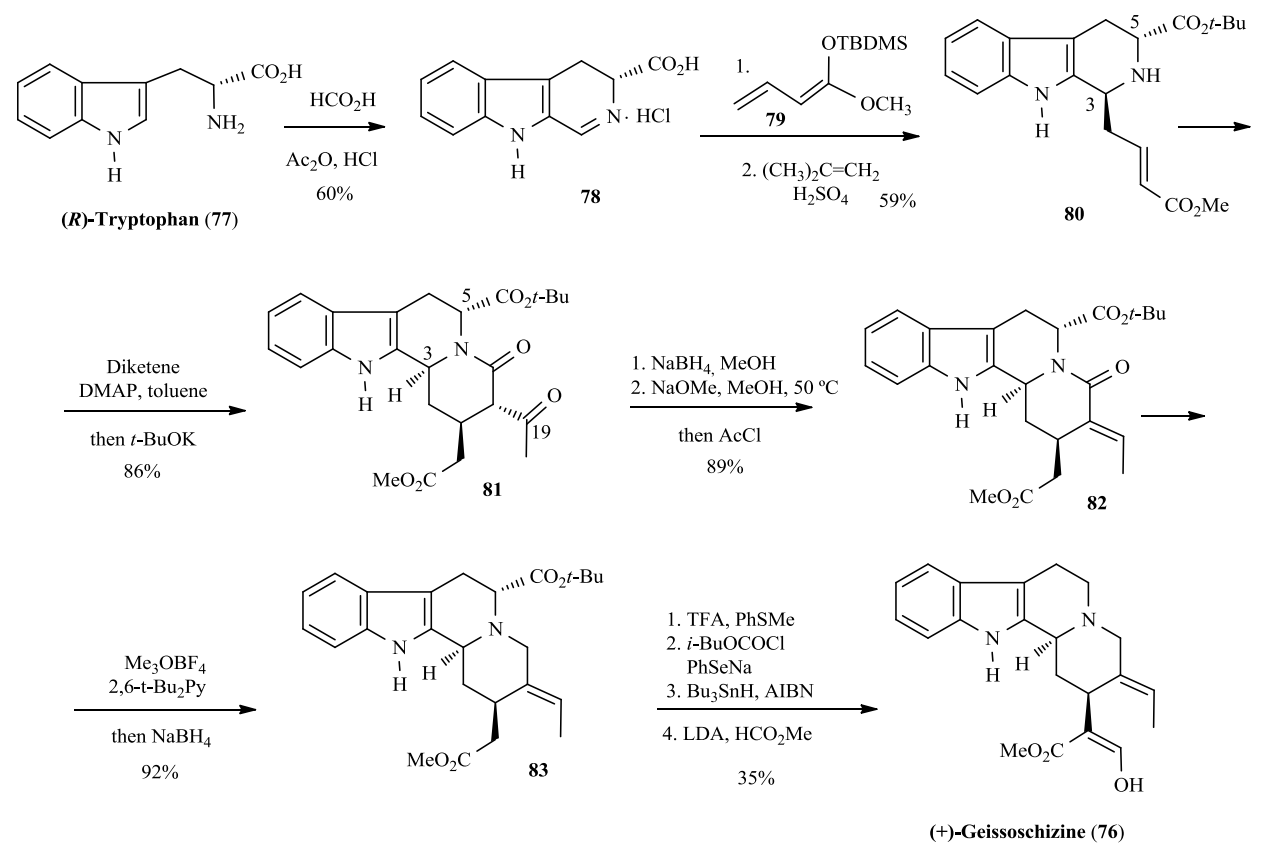

Scheme 3 - Enantioselective synthesis of (+)-geissoschizine (76).

Three years later, this author described the total synthesis of the corynanthe alkaloid $( \pm)$ dihydrocorynantheol (3) and the formal synthesis of ( \pm )-hirsutine (4).[47] Two different 
strategies for assembling the indoloquinolizidine system involving the use of the ring-closing metathesis for the construction of the piperidines D ring, followed by a 1,4-addition to introduce the requisite side chain at C-15, are the key steps of this new racemic approach (Scheme 4).
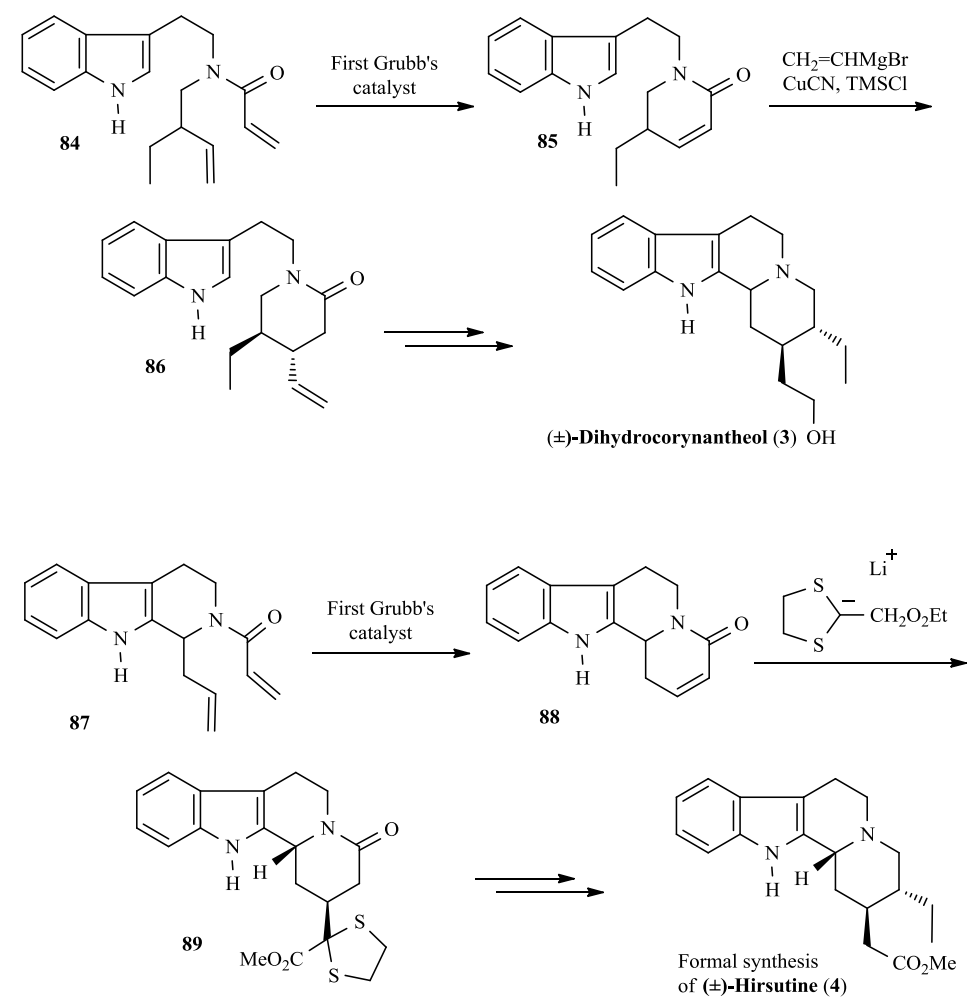

Scheme 4 - Total synthesis of the corynanthe alkaloid ( \pm )-dihydrocorynantheol $(3)$ and the formal synthesis of ( \pm )-hirsutine (4).

\subsubsection{Pictet-Spengler cyclization from a (S)-tryptophanol-derived lactam: Asymmetric} synthesis of both enantiomers of (+)-deplancheine (90)

(+)-Deplancheine (90), an alkaloid with an unusual Corynantheine-type structure, was isolated from the New Caledonian plant Alstonia deplanchei. After its structure elucidation,[48] a number of total syntheses were reported for this alkaloid.[49-52]

In 2005, Steven M. Allin,[51] reported the asymmetric synthesis of both enantiomers of the indole alkaloid deplancheine (Scheme 5) in an approach involving as the key intermediates the tryptophanol-derived lactams $\mathbf{9 3}$, which are easily accessible from $(S)$-tryptophanol (S-91) by a cyclocondensation process with the aldehyde-ester 92. Treatment of the mixture of bicyclic lactams with $2 \mathrm{M} \mathrm{HCl}$ in ethanol led to the formation of the indolo[2,3-a]quinolizine system by 
Pictet-Spengler cyclization, affording $\mathbf{9 4}$ as a single compound. The hydroxymethyl chain underwent cleavage by oxidation to a carboxylic acid derivative through the corresponding aldehyde, followed by the generation of an acyl selenide and subsequent tin-mediated deacylation. The construction of the ethylidene moiety was achieved through a three-step procedure involving generation of the lithium enolate from $\mathbf{9 5}$ and a subsequent aldol reaction with acetaldehyde, activation of the hydroxyl group by mesylation, and finally, DBN-induced elimination to give the target 96. Deprotection of the indole nitrogen atom with TBAF, followed by the reduction of the lactam carbonyl group, as described by Martin and co-workers,[53] satisfactorily afforded (+)-deplanchine (90).

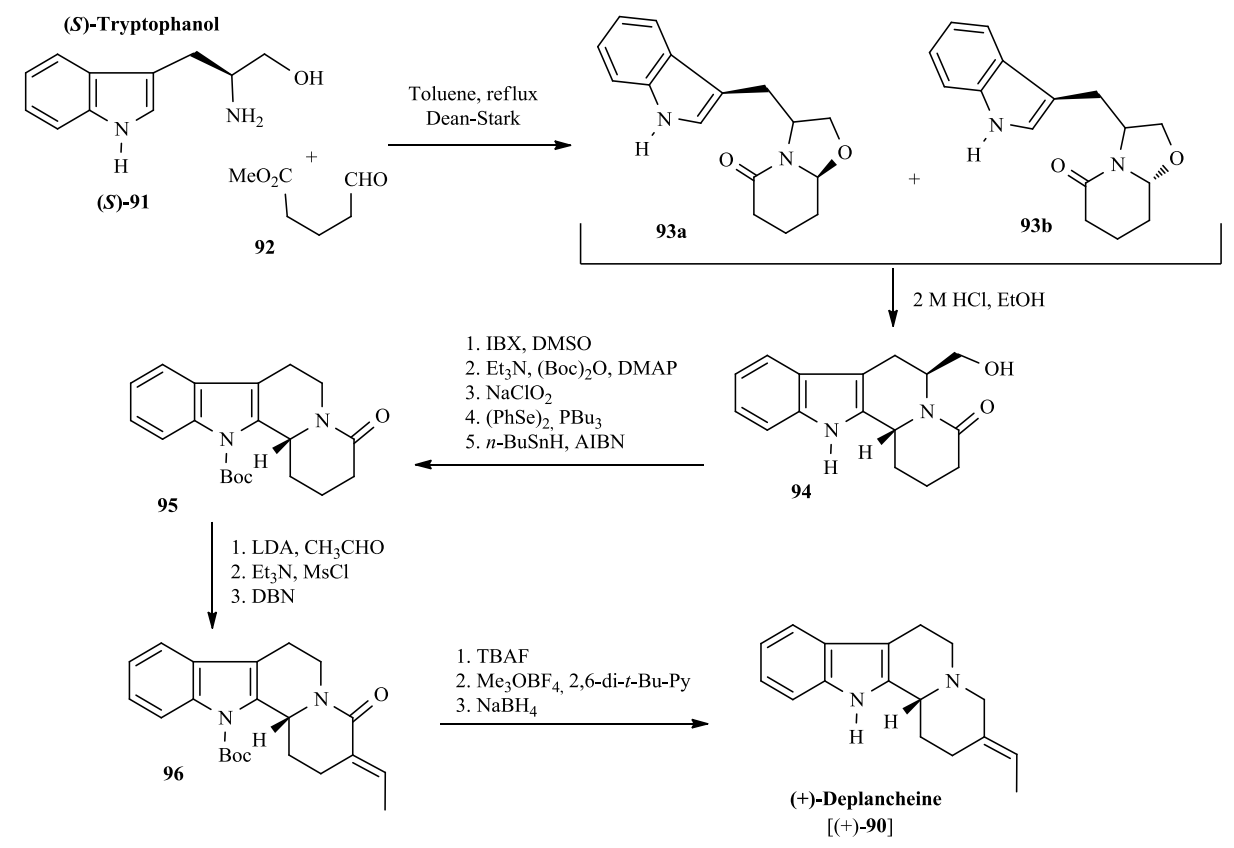

Scheme 5 - Asymmetric synthesis of (+)-deplancheine [(+)-90].

To demonstrate the potential synthetic utility of the methodology, Allin and co-workers have also undertaken an asymmetric synthesis of the enantiomer of the natural product using $(R)$ tryptophanol as the starting material (Scheme 6). 


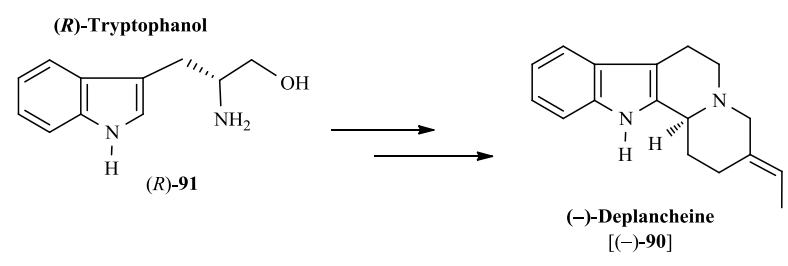

Scheme 6 - Access to (-)-deplancheine $[(-)-90]$.

The stereochemical outcome of the Pictet Spengler cyclizations of tryptophanol-derived lactams was later thoroughly investigated by Amat-Bosch's research group, describing the stereocontrolled generation of $\mathrm{C}-12 \mathrm{~b}$ epimeric indolo[2,3-a]quinolizidine derivatives using the appropriated reactions conditions (Scheme 7).[54-56]

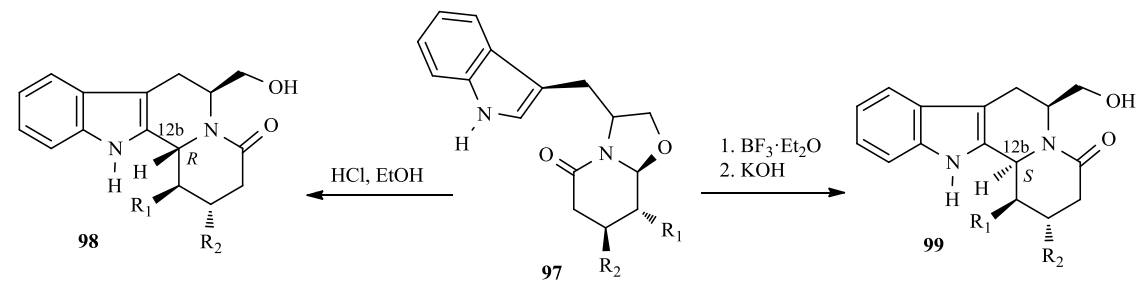

Scheme 7 - Stereocontrolled cyclizations from $(S)$-tryptophanol-derived oxazolopiperidone lactams.

\subsubsection{Modified Bischler-Napieralski reaction from a (S)-tryptophanol-derived lactam:}

Enantioselective formal synthesis of (+)-dihydrocorynantheine (1) and (-)dihydrocorynantheol (3)

Starting from an appropriately substituted tryptophanol-derived lactam bearing an ethyl substituent and an acetate chain at piperidine 3 and 4-positions,[54] respectively, an enantioselective access to indolo[2,3-a]quinolizidine alkaloids (+)-dihydrocorynantheine (1) and (-)-dihydrocorynantheol (3) (Scheme 8) was developed by the Amat/Bosch research group in 2009.[57] The synthesis requires the cyclization at the indole 2-position to take place regioselectively from the lactam carbonyl moiety, which is accomplished using a modified Bischler-Napieralski reaction.

The initial lactam 101 was prepared in $62 \%$ yield by cyclocondensation of $(S)$-tryptophanol $(\mathbf{9 1})$ with racemic $\delta$-oxo diester $\mathbf{1 0 0}$ in a process that involves a dynamic kinetic resolution and the 
differentiation of diastereotopic ester chains, with generation of three stereogenic centers with a well-defined configuration in a single synthetic step. The regio- and stereoselective cyclization on the lactam carbonyl group, leading to an indolo[2,3-a]quinolizidine derivative, took place under modified Bischler-Napieralski conditions. Thus, alkylation of thiolactam (obtained by treatment of lactam $\mathbf{1 0 1}$ with Lawesson's reagent) using benzyl bromide generated a (benzylsulfanyl)-substituted iminium ion, which can be considered as a sulphur analogue of a Bischeler-Napieralski chloro-substituted iminium salt. Sodium borohydride reduction of the iminium intermediate, arising from the subsequent cyclization on the indole 2-position, afforded pentacyclic compound $\mathbf{1 0 2}$ as a single isomer. The indole nitrogen was protected as an $\mathrm{N}$-Boc derivative and the oxazolidine ring of the resulting pentacyclic compound was subjected to reductive ring-opening with borane to give $\mathbf{1 0 3}$. The synthesis of the target alkaloids was completed by cleavage of the hydroxymethyl appendage, which was satisfactorily accomplished by reductive decyanation of an $\alpha$-amino nitrile intermediate. Finally, deprotection of the indole nitrogen led to the tetracyclic ester $\mathbf{1 0 4},[58,59]$ a known synthetic precursor of the alkaloids (+)-dihydrocorynantheine (1) and (-)-dihydrocorynantheol (3).

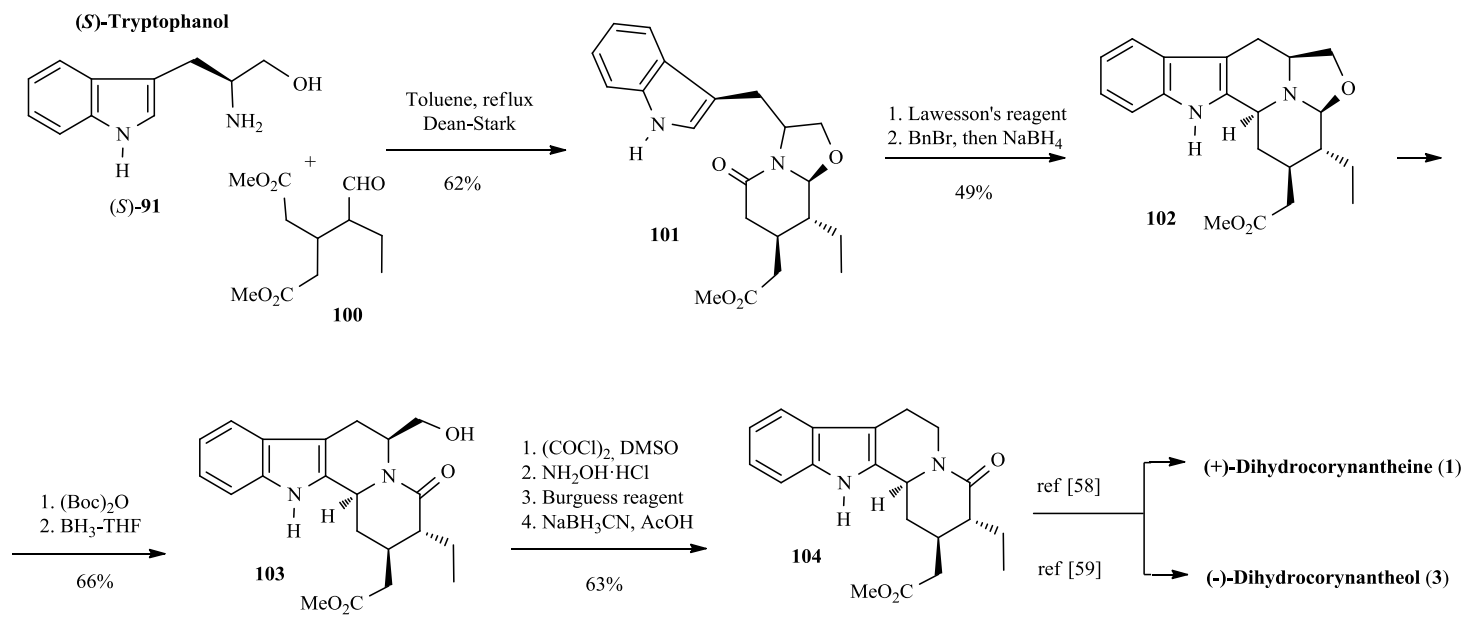

Scheme 8 - Enantioselective formal synthesis of (+)-dihydrocorynantheine (1) and (-)dihydrocorynantheol (3). 


\subsubsection{Access to 9-methoxyindole alkaloids: Total synthesis of 9-methoxygeissoschizol (105), 9-methoxy- $N_{\mathrm{b}}$-methylgeissoschizol (106) and (-)-mitragynine (32).}

Cook, in 2007, described an enantiospecific method for the synthesis of $(R)-4$ methoxytryptophan (107) via a regiospecific Larock heteroannulation. This was a key reaction for the total synthesis of 9-methoxygeissoschizol (105), 9-metroxy- $N_{\mathrm{b}}$-methylgeissoschizol (106), and mitragynine (32) using a asymmetric Pictet-Spengler strategy (Scheme 9).[60]
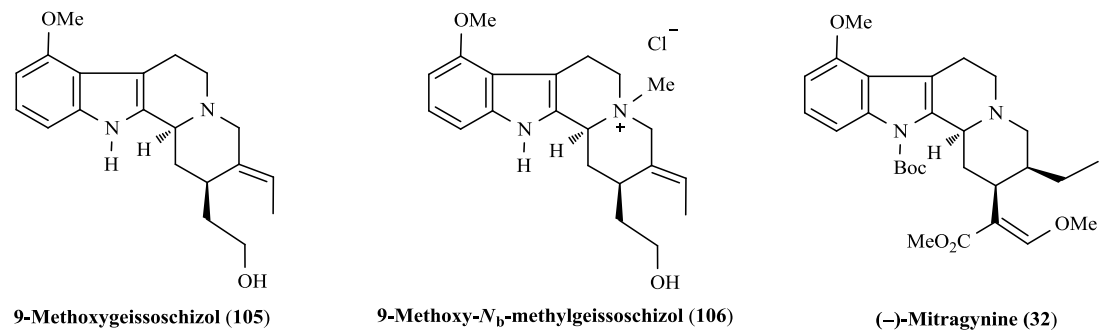

Figure 13 - Chemical structure of 9-methoxygeissoschizol (105), 9-methoxy- $N_{\mathrm{b}^{-}}$ methylgeissoschizol (106) and (-)-mitragynine (32).

Before Cook's synthesis, the preparation of these alkaloids was hampered by the unavailability of 4-methoxytryptophan (107), which could only be obtained in high optical purity using the enzymatic kinetic resolution reported by Ley.[61, 62] The Larock heteroannulation,[63, 64] a palladium-catalysed heteroannulation reaction of substituted $o$-iodoanilines with internal alkynes, is a powerful method for the synthesis of substituted indole derivatives and has been satisfactorily employed for the regiospecific synthesis of both 11- and 12-methoxy-substituted indole alkaloids.[65-68] The strength of the Larock process stems from the regioselectivity that can be achieved when a bulky silyl-substituted alkyne is employed as a substrate. The internal alkyne chosen in this example (109) can be easily prepared by alkylation of Schöllkopf's auxiliary, which in turn is available from L-valine. $[69,70]$ The heteroannulation between 2iodo-3-methoxy- $N$-Boc-aniline 108 and TMS-alkyne 109 gave 4-methoxy- $N_{\mathrm{a}}-\mathrm{H}$ indole 110 in $82 \%$ yield. The subsequent hydrolysis of the Schöllkopf chiral auxiliary was accomplished by concomitant loss of the silyl group of $\mathbf{1 1 0}$, providing 4-methoxy-D-tryptophan ethyl ester $\mathbf{1 1 1}$ in a single step in $91 \%$ yield (Scheme 9). 


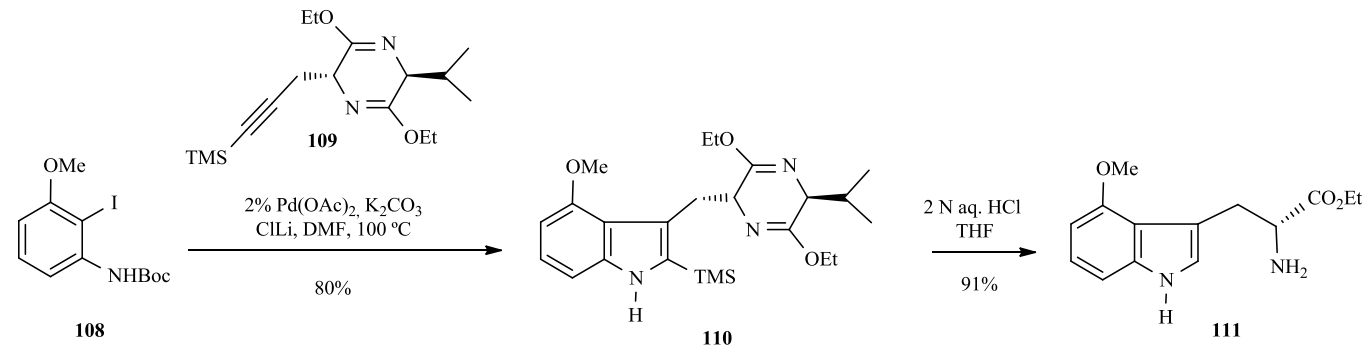

Scheme 9 - Preparation of the 4-methoxy-D-tryptophan ethyl ester 111.

The monoalkylation of benzyl ester 112, obtained by hydrolysis and esterification of 111, with the allylic bromide 113 afforded the secondary amine $\mathbf{1 1 4}$ in $85 \%$ yield. The enantioselective construction of the stereocenter at C-3 was achieved by a modified asymmetric Pictet-Spengler reaction between $\mathbf{1 1 4}$ and the aldehyde $\mathbf{1 1 5}$ to furnish the tetrahydro- $\beta$-carboline system. The $\alpha, \beta$-unsaturated ester moiety was generated via standard transformations, including removal of 1 equivalent of thiophenol, followed by an oxidation and sulfoxide elimination sequence, affording compound 116. The $\alpha, \beta$-unsaturated ester 116 was then subjected to the $\mathrm{Ni}-(\mathrm{COD})_{2^{-}}$ mediated cyclization to provide the desired Corynanthe skeleton 117 in $75 \%$ yield. Removal of the benzyl group was achieved when 117 was treated with $\mathrm{PdCl}_{2}$ in the presence of $\mathrm{Et}_{3} \mathrm{SiH}$ and TEA, and the corresponding carboxylic acid was converted into tetracyclic ester 118 via the Barton-Crich decarboxylation process (Scheme 10).
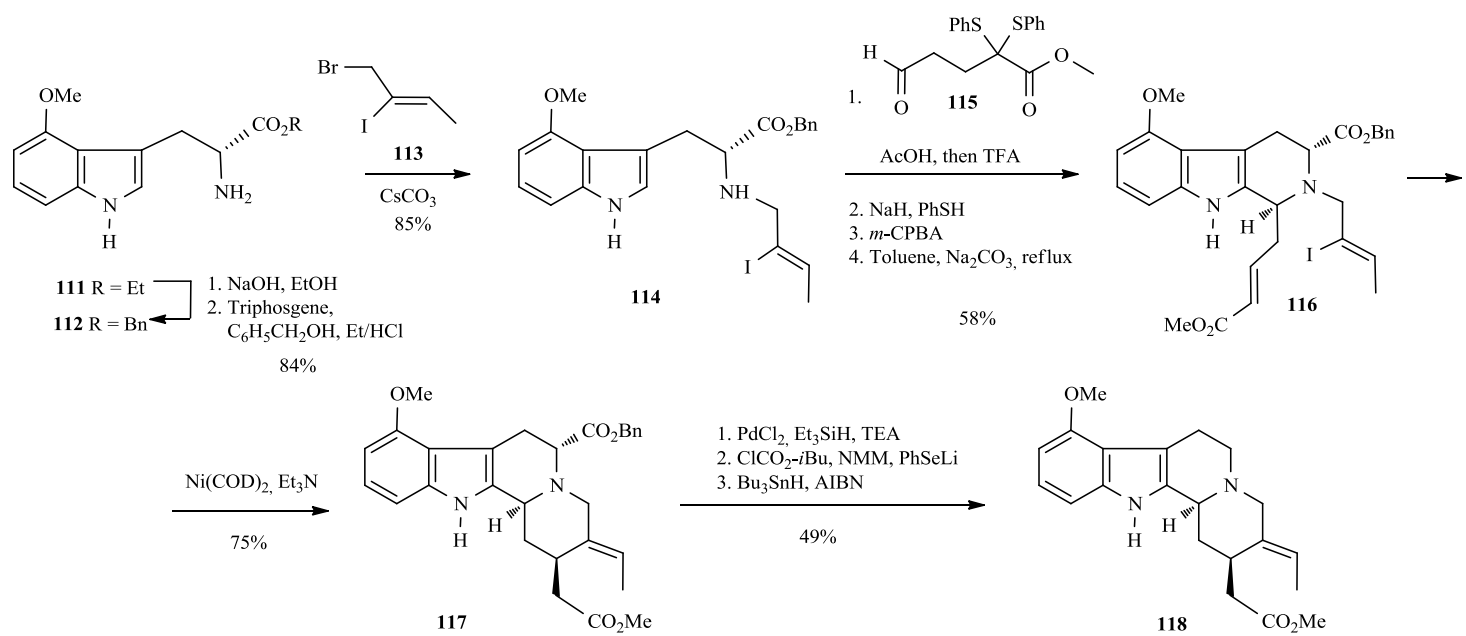

Scheme 10 - Synthesis of the key intermediate 118. 
The ester 118 was envisaged as an advanced precursor for the synthesis of three 9-methoxysubstituted indole alkaloids. Thus, the ester carbonyl moiety present in $\mathbf{1 1 8}$ was reduced with $\mathrm{LiAlH}_{4}$ to give 9-methoxygeissoschizol (105) in 90\% yield, and 9-methoxy- $N_{\mathrm{b}^{-}}$ methylgeissoschizol (106) was then synthesized via $N_{\mathrm{b}}$ methylation with methyl iodide, followed by an iodide exchange using $\mathrm{AgCl}$. To prepare mitraginine (32), reduction of the olefin bond in $\mathbf{1 1 8}$ was required. The reduction of this double bond with Crabtree's catalyst, followed by treatment with (Boc) $)_{2} \mathrm{O}$ in the presence of a catalytic amount of DMAP, provided the Boc derivative 119 in $64 \%$ overall yield. The ester 119 was then subjected to formylation and Boc deprotection. The final acetal formation and $t$-BuOK-mediated elimination of $\mathrm{MeOH}$, analogous to the final steps reported by Takayama,[71] provided (-)-mitragynine (32) (Scheme $11)$.

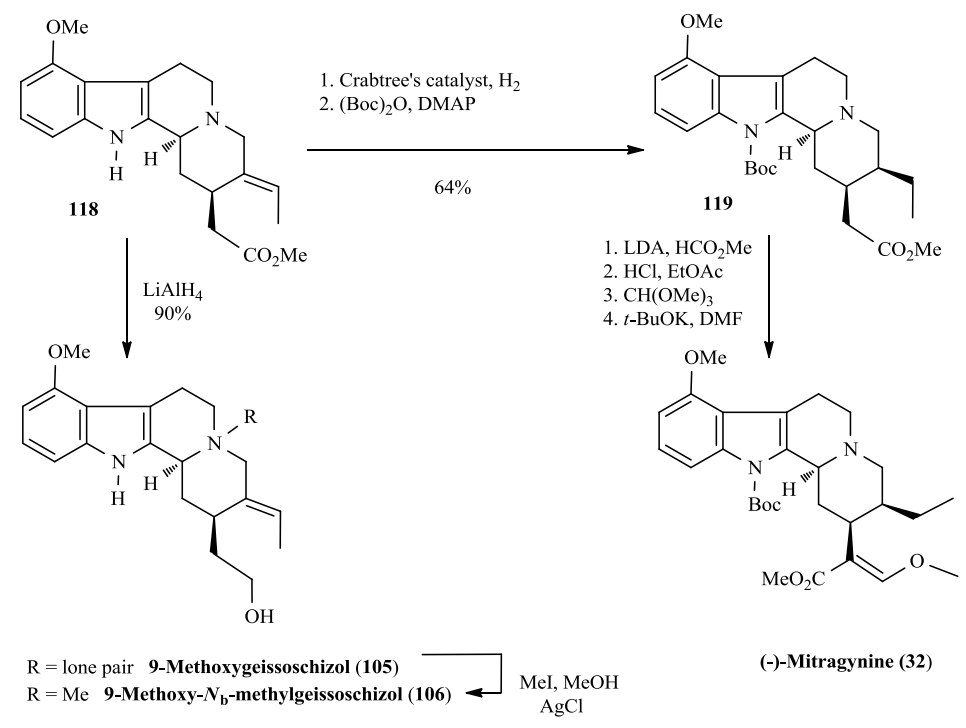

Scheme 11 - Total synthesis of 9-methoxygeissoschizol (105), 9-methoxy- $N_{\mathrm{b}^{-}}$ methylgeissoschizol (106) and (-)-mitragynine (32).

Starting from $(R)$-tryptophan $[(R)-91]$ as a chiral pool reagent, this methodology allows the enantiospecific total synthesis of (-)-corynantheidine (14), (-)-corynantheidol (122), (-)geissoschizol (123), and (+)-geissoschizine (76) via the common key intermediate 119 (Scheme 12).[72] For the construction of the all-D-ring system present in corynantheidol and corynantheine, an intramolecular Heck coupling of $\alpha, \beta$-unsaturated ester 119 and subsequent 
$\mathrm{NaBH}_{4}$-reduction in presence of a catalytic amount of $\mathrm{NiCl}_{2} \cdot 6 \mathrm{H}_{2} \mathrm{O}$ was performed. In turn, to construct the molecular framework of geissoschizol and geissoschizine from 119, a stereoselective Michael reaction consecutively using $\mathrm{Ni}[\mathrm{COD}]_{2}-\mathrm{Et}_{3} \mathrm{~N}$ and $\mathrm{Et}_{3} \mathrm{SiH}$ was employed.

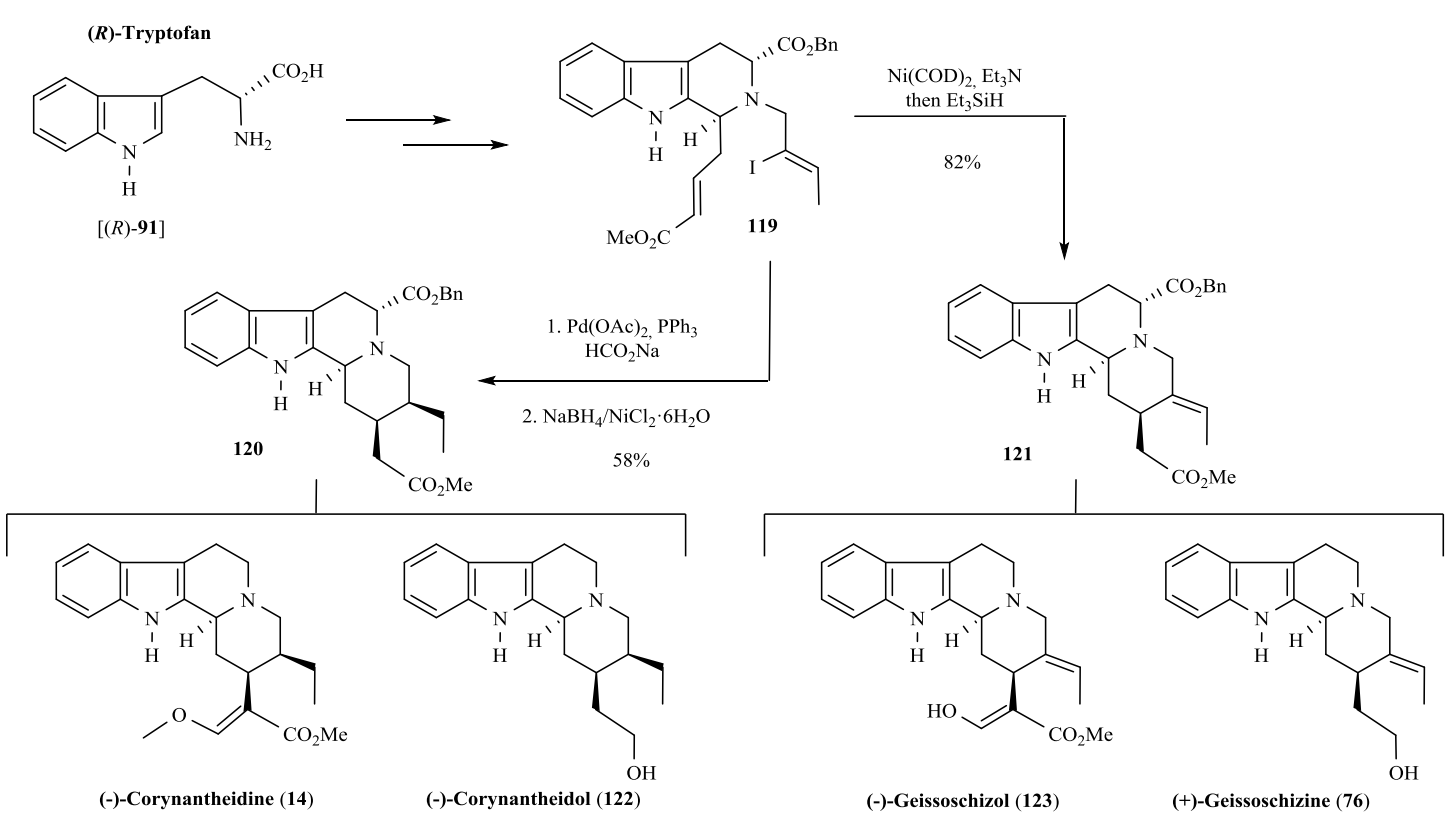

Scheme 12 - Enantiospecific total synthesis of (-)-corynantheidine (14), (-)-corynantheidol (122), (-)-geissoschizol (123), and (+)-geissoschizine (76).

\subsubsection{Regioselective reduction of an imide carbonyl followed by an intramolecular} stereoselective cyclization: Enantioselective formal synthesis of (-)-deplancheine (90).

The enantioselective formal synthesis of (-)-deplancheine $(\mathbf{9 0})$, in a 9-step sequence from glutamic acid in good overall yields and high enantiomeric purity, was recently reported by Argade (Scheme 13).[52]

A carbodiimide-induced coupling reaction of Boc-protected tryptamine $\mathbf{1 2 4}$ with enantiomerically pure $(S)$-tetrahydro-5-oxo-2-furancarboxylic acid (125), prepared from $(S)$ glutamic acid furnished amidolactone $\mathbf{1 2 6}$ in $86 \%$ yield and $96 \%$ enantiomeric purity. Subsequent treatment with $t$-BuOK afforded the base-catalysed rearrangement of $\mathbf{1 2 6}$ to the hydroxyglutarimide $\mathbf{1 2 7}$ in $65 \%$ yield. The hydroxyl group in compound $\mathbf{1 2 7}$ was then transformed to the corresponding acetate using acetic anhydride and triethylamine. The 
regioselective sodium borohydride reduction of the more reactive imide carbonyl group, followed by chemoselective trifluoroacetic acid-induced intramolecular cyclization, afforded indoloquinolicidine derivative $\mathbf{1 2 8}$ as a $23: 2$ mixture of isomers in very good yield. The diastereoisomers $\mathbf{1 2 8} \mathbf{a} / \mathbf{b}$ were quantitatively separated by flash column chromatography and the major isomer (128a) was treated with $\mathrm{K}_{2} \mathrm{CO}_{3} / \mathrm{MeOH}$ to undergo one-pot deacylation and $N$ Boc-deprotection to provide 129 in $92 \%$ yield. Subsequent chemoselective reaction with an excess of phenyl chlorothionoformate in the presence of diisopropylethylamine provided a xanthate intermediate, which was submitted to Barton-McCombie deoxygenation using tributyltin hydride in the presence of AIBN. The resulting lactam 130 was obtained in 54\% yield and 94\% ee (by HPLC) and it was transformed in (-)-deplancheine in a seven-step synthetic sequence.[51]

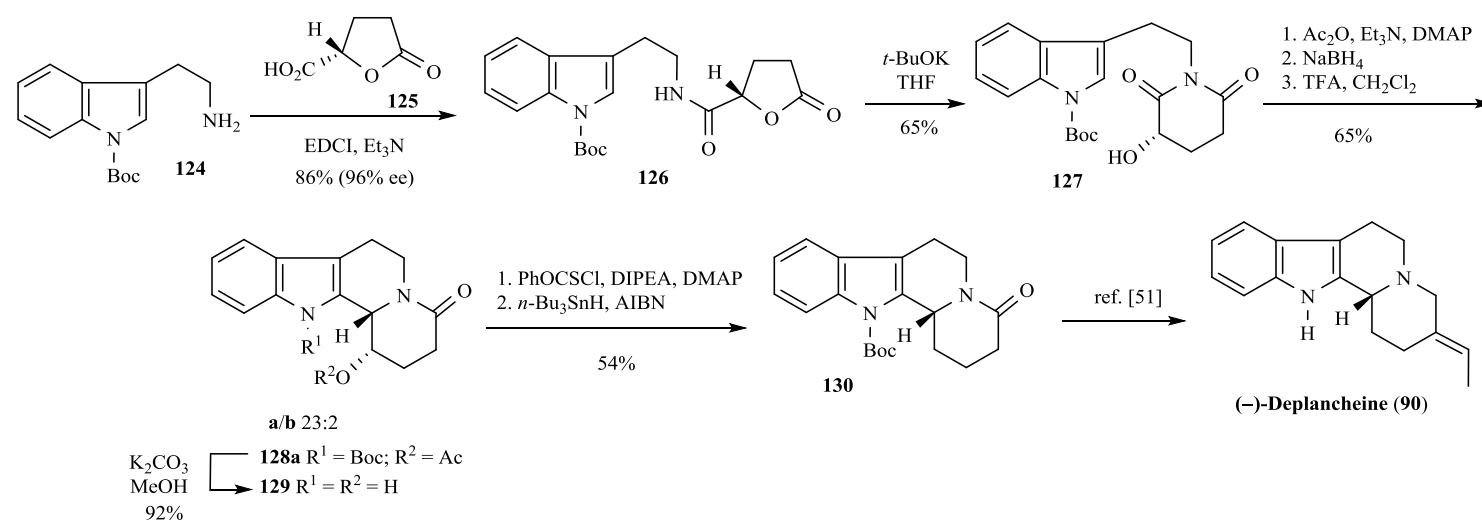

Scheme 13 - Enantioselective formal synthesis of (-)-deplancheine.

\subsection{Non-catalytic cascade/tandem sequences}

Cascade reactions have received considerable attention within organic chemistry, as reflected by the high number of reviews covering this field.[73-75] A variety of terms, including "cascade", "domino", "tandem" and "sequential", are used in the literature, often apparently interchangeably, although efforts have been made to restore order to this area of reaction terminology.[76] Nevertheless, we shall maintain the term used by each author in the original publication. This type of reaction in structurally complex molecule synthesis has several intrinsic advantages: multiple bond formation, time and cost efficiency, atom economy, 
environmental sustainability as well as applicability to diversity-oriented high-throughput synthesis. Since the synthetic effort toward natural products and other interesting compounds usually requires the introduction of several stereogenic centers, the design of cascades to provide specific targeted molecules of structural and stereochemical complexity constitutes a significant intellectual challenge.

\subsubsection{Stereoselective addition of a chiral $\alpha$-sulfinyl ketamine anion to methyl acrylate:} Asymmetric synthesis of 1,2,3,4,6,7,12,12b-octahydroindolo[2,3-a]quinolizidine (131).

One of the first examples of enantioselective synthesis of indoloquinolizidine alkaloids using a tandem reaction was reported by Hua in 1991, and it was based on the asymmetric 1,4addition/ring-closure procedure (Scheme 14).[77]

The chiral sulfinyl ketimine 134 was prepared from harmalan (132) and (-)-menthyl $p$ toluenesulfinate (133), and subsequently used in the stereoselective conjugate addition with methyl acrylate, followed by in situ cyclization. The resulting lactam $\mathbf{1 3 5}$, formed in $77 \%$ yield, was then submitted to $\mathrm{NaCNBH}_{3}$-reduction of the double bond. In contrast with previous results of this author, the reduction in this case was not stereoselective and a 1.9:1 mixture of diastereomers was formed. After separation in column chromatography, desulfuration of $\mathbf{1 3 6 \mathbf { b }}$ with Raney Nickel, followed by reduction with $\mathrm{LiAlH}_{4}$, afforded $1,2,3,4,6,7,12,12 \mathrm{~b}$ octahydroindolo[2,3-a]quinolizidine (131).

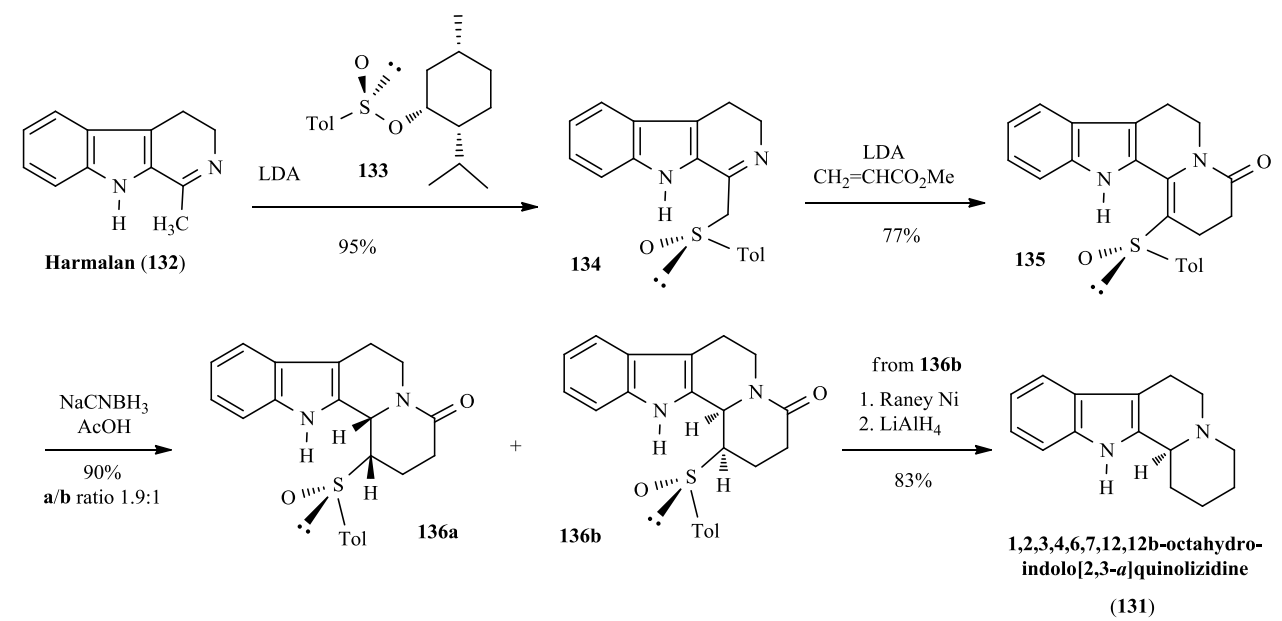


Scheme 14 - Asymmetric synthesis of 1,2,3,4,6,7,12,12b-octahydroindolo[2,3-a]quinolizidine

(131).

\subsubsection{A domino Knoevenagel-hetero-Diels-Alder reaction strategy: Enantioselective total} synthesis of (+)-hirsutine (4) and (+)-dihydrocorynantheine (1)

The enantioselective synthesis of corynanthe indole alkaloids (+)-hirsutine (4) and (+)dihydrocorynantheine (1) by a domino Knoevenagel-hetero-Diels-Alder reaction, using enantiomerically pure tetrahydro- $\beta$-carboline carbaldehydes as starting material, was described in 1999 by Tietze.[78]

Aldehydes $140\left(N_{\text {ind }}-\right.$ Boc $)$ and $141\left(N_{\text {ind }}-\mathrm{H}\right)$ were prepared on a large scale by separation of the diastereomeric amides 138, which were prepared from rac-137 and (-)-camphanic acid as shown in scheme 15.

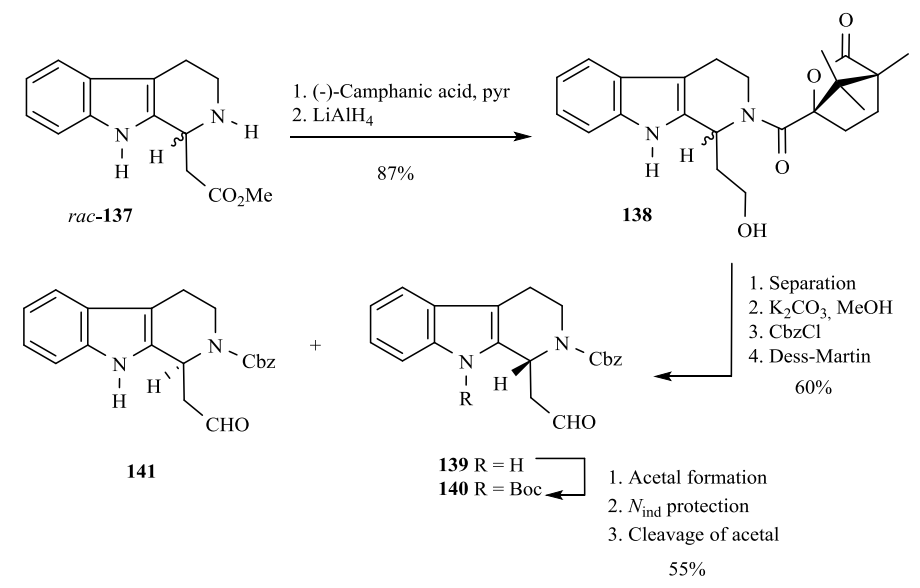

Scheme 15 - Preparation of chiral non-racemic tetrahydro- $\beta$-carboline carbaldehydes 140 and 141.

Condensation of the aldehyde 140 with Meldrum's acid (142) and 4-methoxybenzyl butenyl ether 143, in an ultrasonic bath and in the presence of ethylenediamine diacetate (EDDA), led to cycloadduct 145 in $84 \%$ yield and with an asymmetric induction greater than 20:1. Direct solvolysis without further purification, followed by hydrogenation, afforded the tetracycle $\mathbf{1 4 8 a}$ in $67 \%$ yield as a single product. 
Similarly, the domino Knoevenagel-hetero-Diels-Alder reaction of 141 with 142 and the enol ether 144 gave the diastereomeric cycloadducts 146 in very good yield, although with somewhat lower asymmetric induction of 4.8:1. The mixture of cycloadducts was converted into the tertbutoxycarbonyl derivatives 147, which were then treated with methanol/ $\mathrm{K}_{2} \mathrm{CO}_{3}$ and hydrogenated. Chromatographic separation gave the enantiomerically pure diastereomer $\mathbf{1 4 8 b}$ in $62 \%$ overall yield from 147. Cleavage of the tert-butoxycarbonyl group in 148a and $\mathbf{1 4 8 b}$, followed by condensation with methyl formate and treatment with diazomethane by known procedures,[71] gave the desired enantiomerically pure indole alkaloids (+)-hirsutine (4) and (+)-dihydrocorynantheine (1) (Scheme 16).

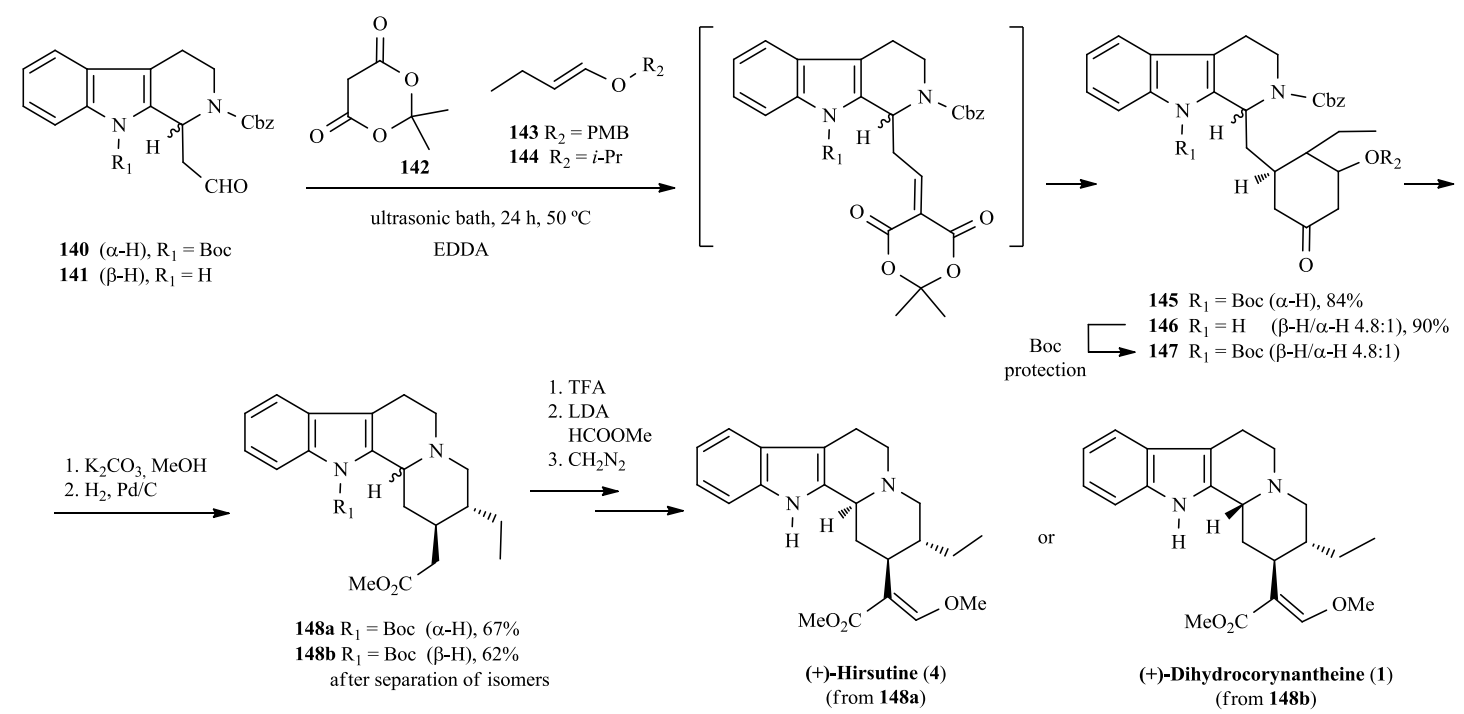

Scheme 16 - Enantioselective total synthesis of (+)-hirsutine (4) and (+)-dihydrocorynantheine

(1).

\subsubsection{Tandem retro-aldol-Pictet-Spengler: Total synthesis of (-)-dihydrocorynantheol (3)}

The diastereocontrolled synthesis of the (-)-dihydrocorynantheol (3) alkaloid was accomplished by Ogasawara and Iwabuchi[79] employing a cascade sequence consisting of a Brønsted-acidpromoted retro-aldol reaction of the key intermediate $\mathbf{1 5 3}$ and a subsequent stereocontrolled Pictet-Spengler reaction.

Amide 153 was prepared in $62 \%$ overall yield (Scheme 17) from the bicyclo[3.2.1]octenone chiral building block (-)-149[80] in a four-step sequence involving the regioselective and 
convex-face selective introduction of a nitromethyl group, ketalization under Noyori's conditions,[81] reduction of the nitro group to the primary amine, and condensation with indole3-acetic acid (152).
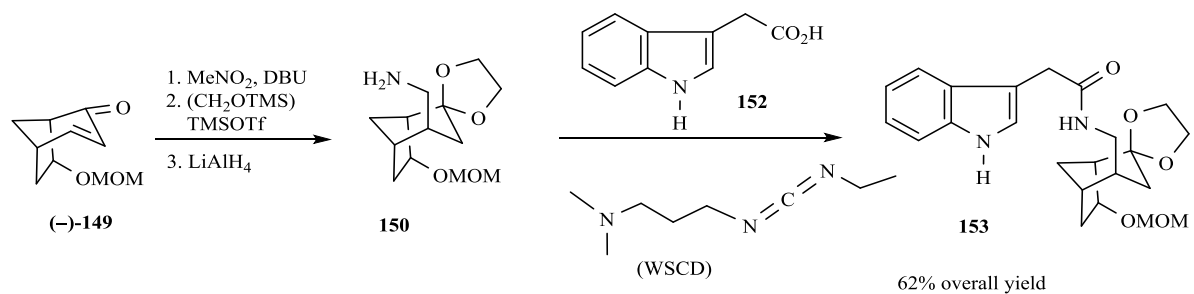

Scheme 17 - Preparation of the key intermediate 153.

The treatment of amide $\mathbf{1 5 3}$ with methanesulfonic acid in boiling dioxane afforded a yohimbinetype derivative 154a in 52\% yield, together with pseudoyohimbane (154b) in $23 \%$ yield, by a tandem retro-aldol-Pictet-Spengler reaction and C-3 epimerization. The recovered $\mathbf{1 5 4 b}$ was subjected to the above-mentioned acidic conditions to obtain 154a in 59\% yield and unreacted 154b in $29 \%$ yield. For the transformation of the E-ring moiety to the seco form present in the dihydrocorynantheol (3) alkaloid, the ketal moiety was deprotected, and the resulting ketone was reacted with pyrrolidine to form an enamine, which was immediately treated with trimethylene dithiotosylate to generate $\mathbf{1 5 5}$. Upon exposure to potassium hydroxide in boiling tert-butyl alcohol, the dithioketone moiety was cleaved and the resulting acid was esterified to obtain methyl ester 156. Treatment of this compound with iodomethane in aqueous acetonitrile, followed by the reduction of the resulting aldehyde with sodium borohydride, afforded the corresponding alcohol, which was protected as TBS ether to give compound 157. After subsequent treatment with lithium aluminium hydride and mesylation, the resulting mesylate was subjected to reduction using $\mathrm{LiAlH}_{4}$ in boiling dioxane to furnish, with the concomitant reduction of the amide moiety, as well as deprotection of the TBS group, completed the synthesis of the target alkaloid (-)-dihydrocorynantheol (3) (Scheme 18). 


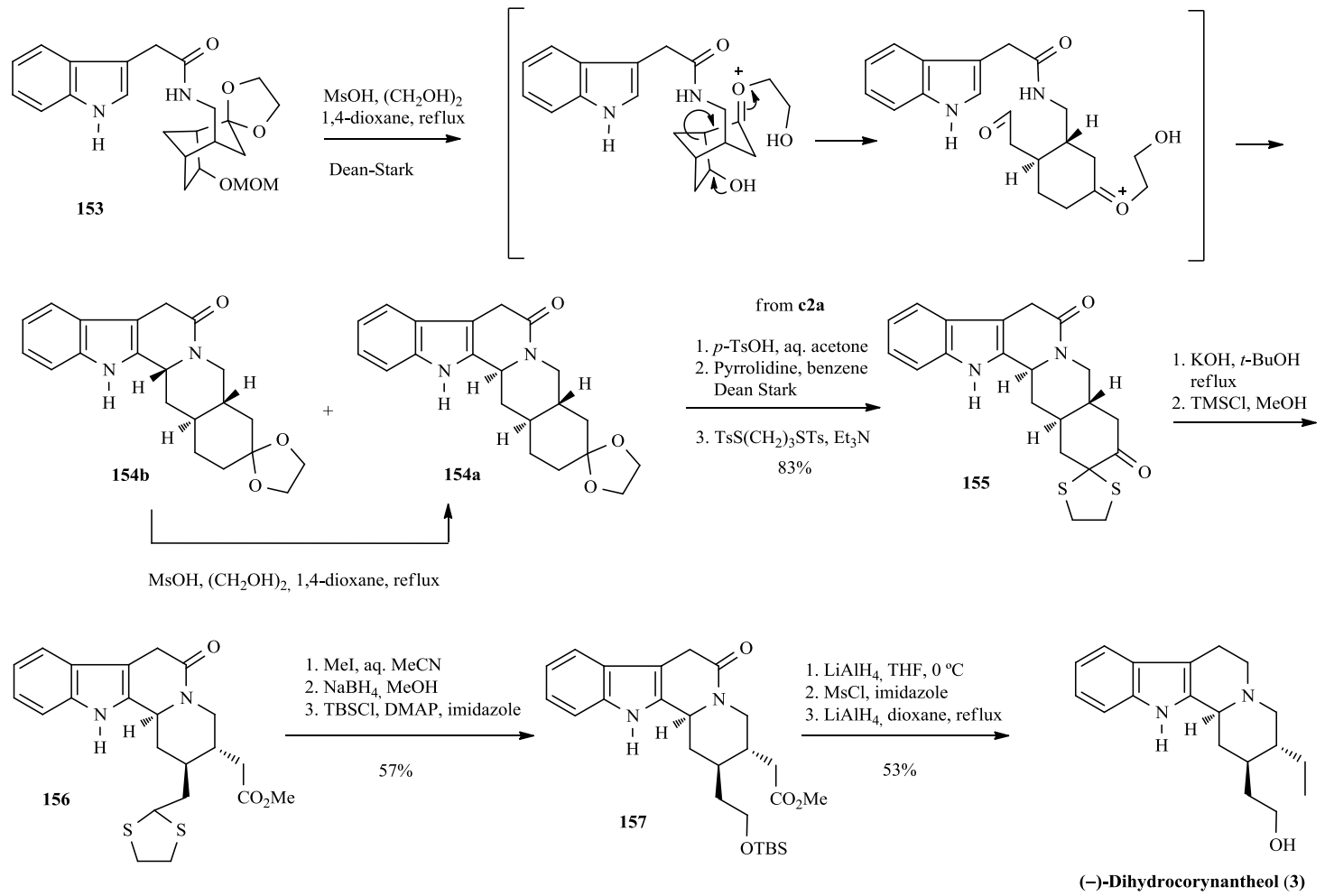

Scheme 18 - Total synthesis of (-)-dihydrocorynantheol (3).

\subsubsection{One-pot asymmetric azaelectrocyclization: Enantioselective synthesis of (-)-} corynantheidol (122) and formal synthesis of (-)-corynantheidine (14).

Soon after the publication of the one-pot asymmetric azaelectrocyclization procedure for the synthesis of chiral 2,4,5-trisubstituted tetrahydropyridines,[82] Katsumura demonstrated the usefulness of his methodology with an enantioselective synthesis of the indole alkaloids (-)corynantheidol (122) and (-)-corynantheidine (14).[83]

The one-pot azaelectrocyclization requires an initial mixture of the tetrasubstituted vinyl iodide 158 and the aminoindanol derivative 159, and the subsequent addition of indolyl vinyl stannane 160, and catalytic amounts of $\mathrm{Pd}_{2}(\mathrm{dba})_{3}$, trifurylphosphine and $\mathrm{LiCl}$. Four new bonds and three stereocenters were generated in a single operation, affording aminoacetal $\mathbf{1 6 1}$ as a single diastereomer in $77 \%$ yield (Scheme 19). 

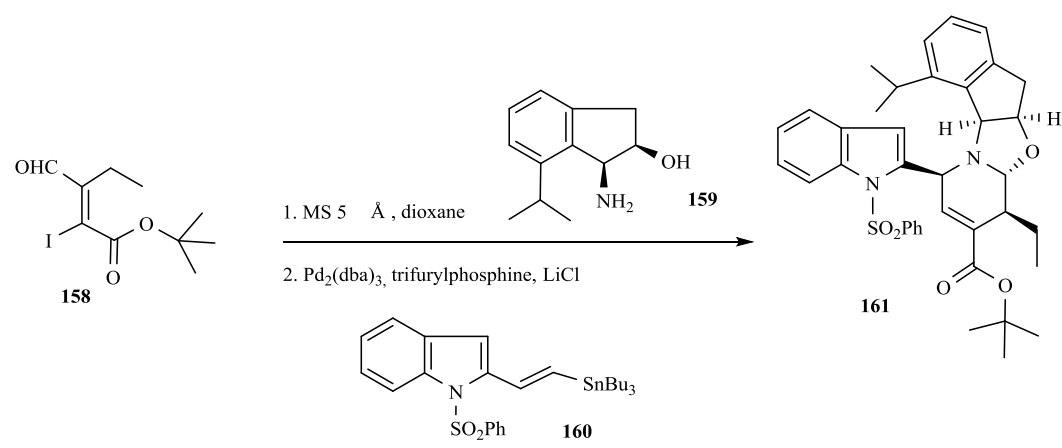

Scheme 19 - Preparation of the chiral non-racemic piperidine 161.

The reduction of the ester group and aminoacetal moiety with DIBAL-H, followed by conversion of the resulting alcohol into a carbonate moiety, allowed the one-carbon elongation at the four position of the tetrahydropiridine ring by $\mathrm{CO}$ insertion, affording the ester $\mathbf{1 6 3}$. Elimination of the indanol chiral auxiliary was accomplished by oxidation with lead tetraacetate in the presence of $n$-propylamine, leading to the corresponding secondary amine. The tetracyclic ring system was constructed from the amine intermediate by Bosch's Pummerer cyclization sequence,[84] via the generation of sulfoxide intermediate 164 and subsequent cyclization with trimethylsilyl triflate in the presence of diisopropylethylamine. The removal of the resulting thiophenyl substituent and the phenylsulfonyl group at the indole nitrogen in $\mathbf{1 6 5}$ was carried out using the Birch reduction, and the stereoselective reduction of the double bond in the tetrahydropyridine ring was accomplished by hydrogenation with platinum dioxide, affording (-)-corynantheidol (122) (Scheme 20).

The formal synthesis of (-)-corynantheidine (14) from the intermediate 165 was achieved by $\mathrm{Ba}(\mathrm{OH})_{2}$ hydrolysis of the ester moiety to afford carboxylic acid 166. The subsequent Birch reduction to remove the thiophenyl and benzenesulfonyl groups, esterification, and catalytic hydrogenation provided Cook's intermediate 167, from which the synthesis of (-)corynantheidine (14) could be completed in two steps (Scheme 20).[72] 

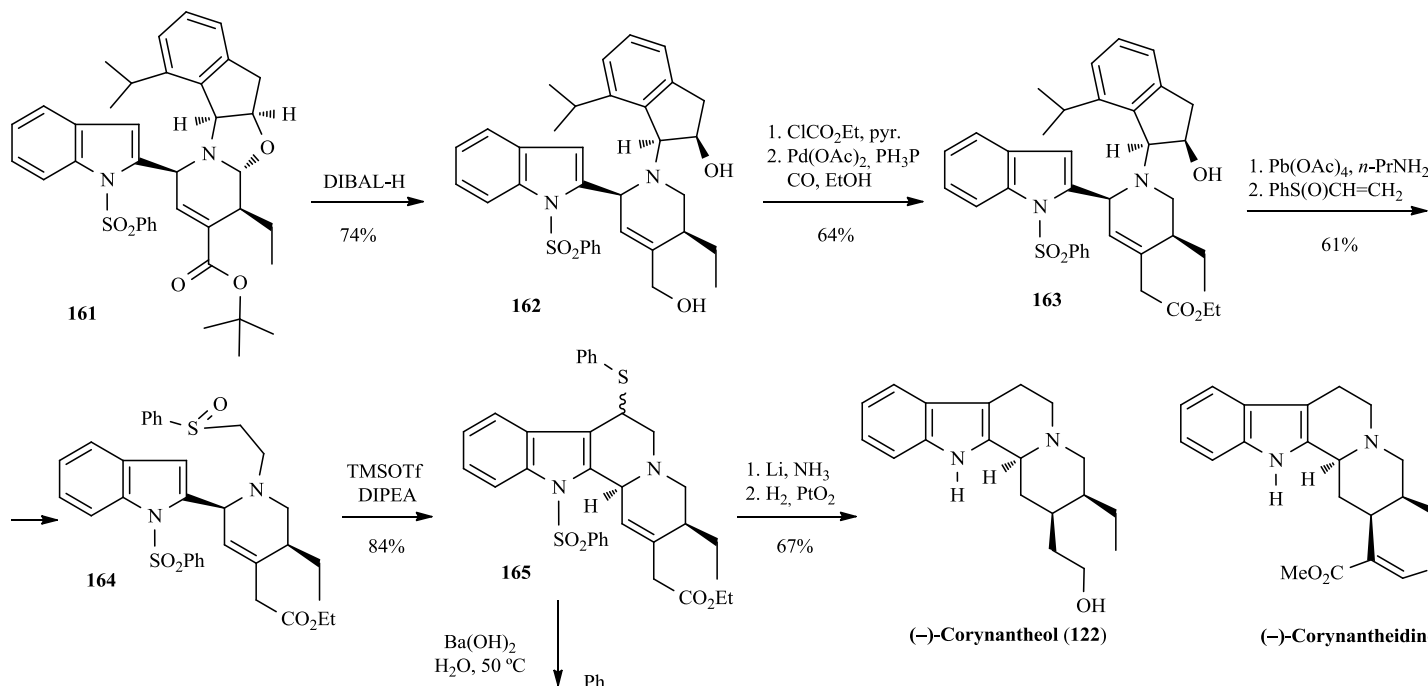

(-)-Corynantheol (122)

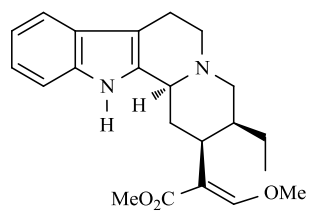

$100 \%$
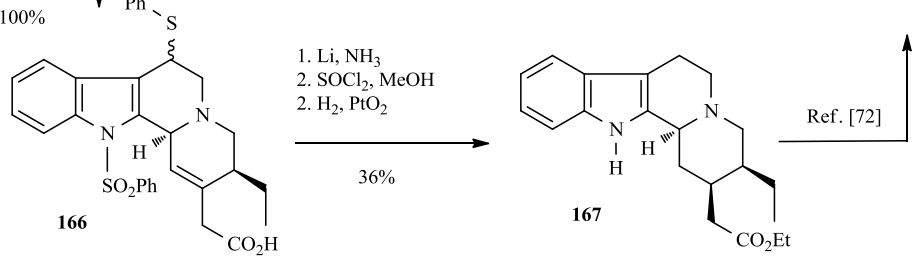

Scheme 20 - Enantioselective synthesis of (-)-corynantheidol (122) and formal synthesis of (-)corynantheidine (14).

2.2.5 Stereodivergent synthesis of $( \pm)$-geissoschizol (123), $( \pm)$-corynantheidol (122), $( \pm)$ dihydrocorynantheol (3) from a single synthetic intermediate.

Traditionally, indoloquinolizidine alkaloids have been approached by individual strategies that have given access to a small number of structurally similar synthetic targets. In 2010, Robert M. Williams envisioned a approach based on a large-scale synthesis of a functionalized lactone intermediate 171, via a tandem Michael cyclization and Horner-Wadsworth-Emmons olefination, which can be rapidly modified to obtain different alkaloids of this group, as well as secologanin dopamine alkaloids.[85]

Assembly of lactone 171 (Scheme 21) [86] begins with the condensation of aldehyde $\mathbf{1 6 8}$ with commercially available trimethyl acetophosphonate under Masamune-Roush conditions, affording the unsaturated ester $\mathbf{1 6 9}$ as a single isomer in good yield. Deprotection and subsequent esterification gave the desired substrate 170, which upon treatment with $\mathrm{Cs}_{2} \mathrm{CO}_{3}$ and 
acetaldehyde underwent sequential intramolecular Michael cyclization and HWE-olefination to yield lactone $\mathbf{1 7 1}$ as a 1.6:1 mixture of $E / Z$ isomers.

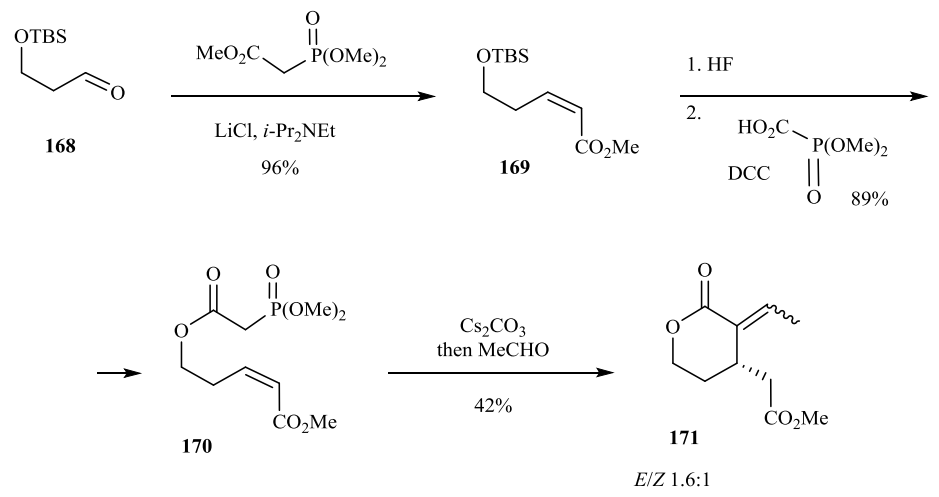

Scheme 21 - Preparation of the intermediate 171.

Reduction of the lactone carbonyl of $\mathbf{1 7 1}$ with DIBAL-H, followed by a one-step reductive amination/lactam cyclization with tryptamine, afforded lactam intermediate 172. Protection of the hydroxyl functionality as an acetate ester, followed by Bischler-Napieralski cyclization, gave acetate 173, which was deprotected to yield ( \pm )-geissoschizol in good yield (Scheme 22). Alternatively, catalytic hydrogenation of the olefin of lactone $\mathbf{1 7 1}$ exclusively generates the corresponding cis- lactone 174a, presumably due to the delivery of hydrogen to the least sterically hindered face of the olefin. Conversely, conjugate reduction leads solely to the thermodynamically favored trans-isomer of the disubstituted lactone 174b. Following a sequence similar to the above, corynantheidol and dihydrocorynantheol (3) were obtained from lactones 174a and 174b, respectively (Scheme 22). However, pure lactone 174b led to a 1.3:1 mixture of isomers, which were separated by column chromatography after the formation of the indoloquinolizidine system. 


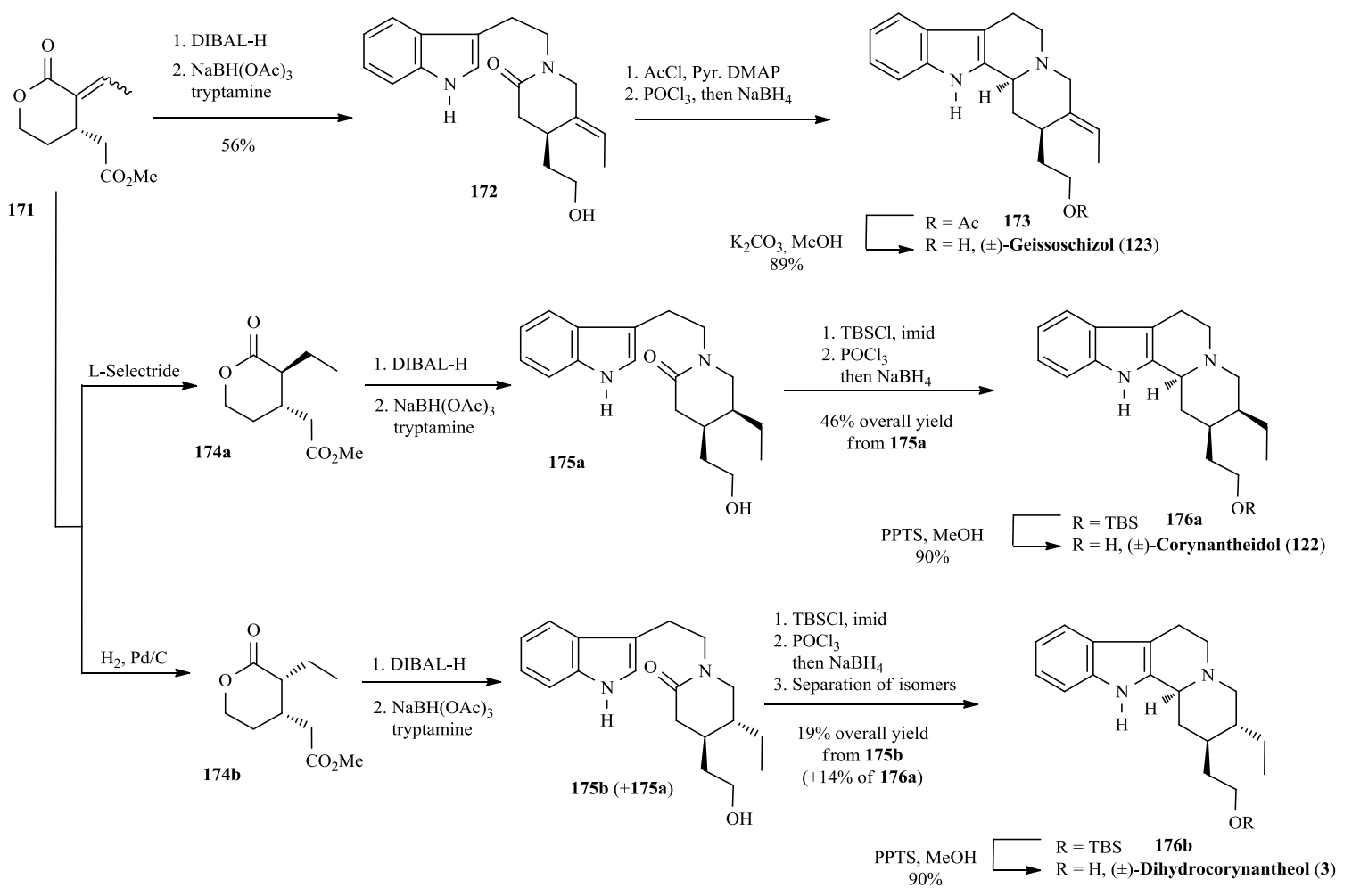

Scheme 22 - Stereodivergent synthesis of $( \pm)$-geisssoschizol (123), ( \pm )-corynantheidol (122),

( \pm )-dihydrocorynantheol (3).

\subsection{Asymmetric metalcatalysis/organocatalysis approaches}

Catalysis has grown to play a prominent role in chemistry as it enables compounds to be prepared efficiently with atom economy. As well as producing far less waste is produced, new selective catalytic processes facilitate short-cuts in total synthesis. In recent years, asymmetric syntheses using organic compounds as catalysts have attracted considerable attention [87] as they are environmentally friendly compared with conventional transition metal catalysts. However, although this area is under intense study, there are still few reports [88, 89] on the application of this methodology for asymmetric total synthesis of natural products.

2.3.1 Asymmetric hydrogen-transfer reaction: Enantioselective Total synthesis of (+)arborescidine A 
A short enantiocontrolled syntheses of (+)-arborescidine A (13), by taking advantage of the Noyori asymmetric hydrogen-transfer reaction of appropriately functionalized $\beta$-carboline derivative, was described by Rawal in 2004 (Scheme 23).[90]

The 6-bromotryptamine (178), which was prepared from 6-bromoindole (177) following the Shumaker and Davidson method,[91] reacted with glutamic anhydride affording the corresponding amide carboxylic acid, which was then esterificated. Subsequent treatment with $\mathrm{POCl}_{3}$ promoted the Bischler-Napieralsky cyclization to produce imine $\mathbf{1 8 0}$ in $86 \%$ yield. The Noyori reduction[92, 93] of imine 180, to set the required asymmetry, was accomplished with preformed $(S, S)$-TsDPEN-Ru(II) complex, which afforded, after in situ cyclization, lactam $\mathbf{1 8 1}$ in $89 \%$ yield and $96 \%$ ee. Finally, alane reduction of the lactam carbonyl afforded (+)arborescidine A (13).
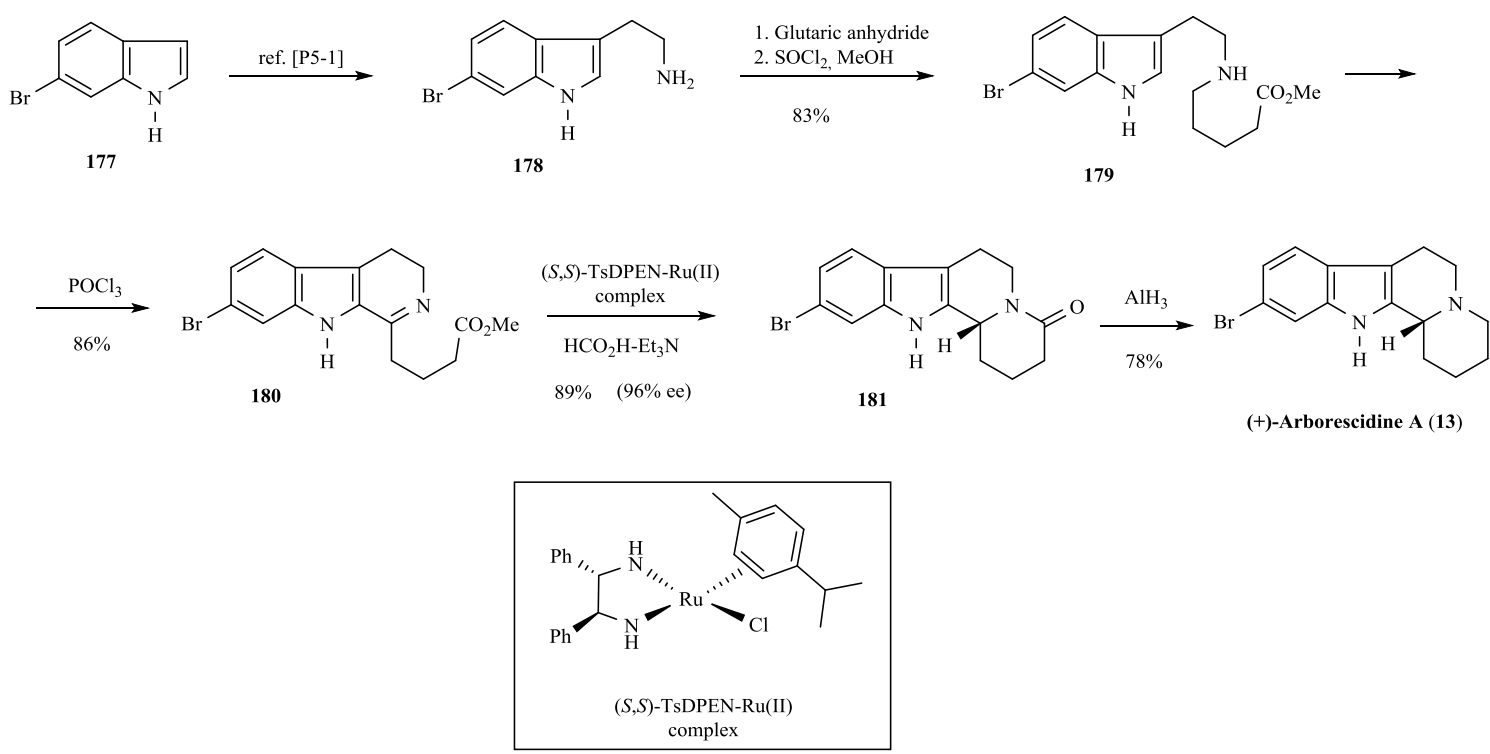

Scheme 23 - Enantioselective total synthesis of (+)-arborescidine A (13).

\subsubsection{A Stereodivergent Strategy for the Preparation of Corynantheine alkaloids}

A general and excellent catalytic asymmetric strategy for the total and formal synthesis of a broad number of optically active natural products from the corynantheine and ipecac alkaloid families, have been described by Franzén in 2011.[94] The construction of the core alkaloid skeletons, with the correct absolute and relative stereochemistry, relies on an enantioselective 
and diastereodivergent one-pot cascade sequence followed by an additional diastereodivergent reaction step. Starting from common and easily accessible starting materials and using a common synthetic route this approach gives access to the total synthesis of the indolo[2,3a]quinolizidine alkaloids (-)-dihydrocorynantheol (3), (+)-hirsutinol (182), (-)-corynantheol (183), (-)-dihydrocorynantheal (184), and (-)-corynantheal (185).

The one-pot stereoselective construction of the quinolizidine carbon skeleton required the $\alpha, \beta$ unsaturated aldehyde 186, which was easily accessible through the cross-metathesis of acroleine and 3-butenol, and the $\beta$-ketoamides $\mathbf{1 8 7}$ which was obtained through the condensation of tertbutyl acetoacetate with the corresponding 2-arylethanamine. In the presence of catalyst $\mathbf{1 8 8}, \beta$ ketoamides 187 smoothly reacted with the $\alpha, \beta$-unsaturated aldehyde to give a diastereomeric mixture of lactols intermediates and then the reaction was quenched by addition of trifluoroacetic acid (TFA) giving a 1:1 mixture of the two ring-junction isomers $\alpha-189$ and $\beta$ 189, which could be isolated in good yields and high enantioselectivity in a two-step one-pot process. Interestingly, when the reaction was quenched with acetyl chloride resulted in the formation of the thermodynamically favored indolo[2,3-a]quinolizidine $\alpha-\mathbf{1 8 9}$ as the only observable isomer, whereas when using benzoyl chloride, a switch in diastereoselectivity it happened and the kinetically favored product $\beta \mathbf{- 1 8 9}$ was obtained as the major isomer in 82:18 diastereoselectivity. It is also worth noting that the kinetically favored $\beta$-indolo[2,3a]quinolizidine $\beta-\mathbf{1 8 9}$ could be epimerized to the thermodynamically favored $\alpha-\mathbf{1 8 9}$ epimer by treatment with TFA heated at reflux to give a ratio of 85:15 in favor of the $\alpha$-epimer. Subsequent reduction of the crude reaction mixture from the one-pot cascade was accomplished by initial alkylation with triethyloxonium tetrafluoroborate, followed by $\mathrm{NaBH}_{4}$-reduction to give the corresponding amines $\mathbf{1 9 0}$ in high to moderate overall yields, and at this stage, the $\alpha-$ and $\beta$-epimers could be easily separated by flash column (Scheme 24). 


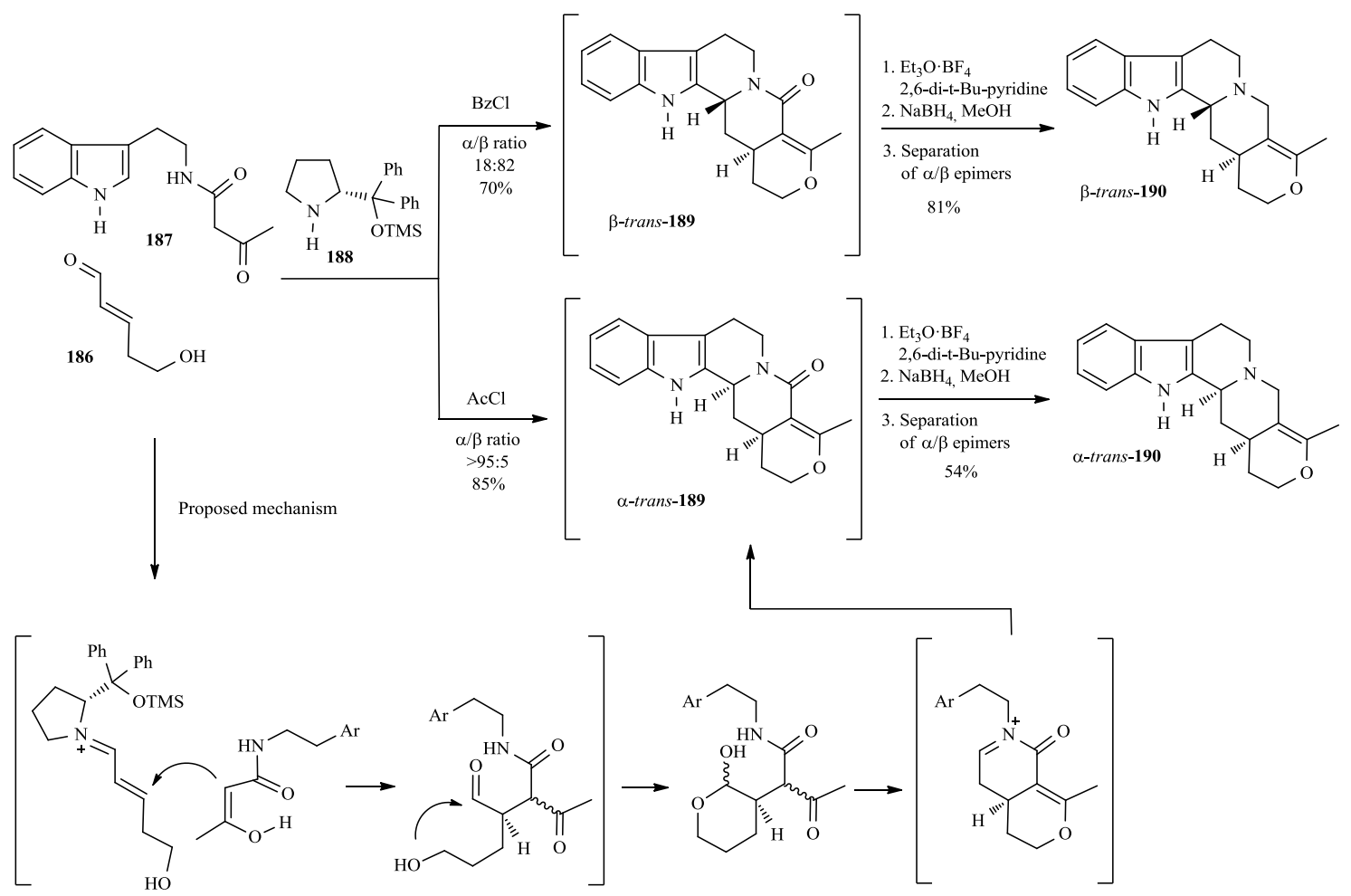

Scheme 24 - Preparation of the $\alpha$ - and $\beta$-epimers of the amine 190.

Subsequent treatment of the $\alpha$-epimer $\alpha-\mathbf{1 9 0}$ with $\mathrm{HCl}$ in water/THF at room temperature gave exclusively the trans ring junction of the corresponding lactol $\alpha$-trans-191. The selective formation of this stereocenter is due to the thermodynamic stability of the all-equatorial quinolizidine structure, and attempts to access the $\alpha$-cis configuration have failed. However, in the $\beta$-series treatment of indolo[2,3-a]quinolizidine with $\mathrm{HCl}$ in water/THF at room temperature gave a 4:1 mixture of $\beta$-cis-191 and $\beta$-trans-191. Interestingly, lactol formation at elevated temperatures $\left(\mathrm{HCl}\right.$ in water/THF at $\left.65^{\circ} \mathrm{C}\right)$ reversed the selectivity and $\beta$-trans-191 was formed with a 5:1 diastereomeric ratio. The resulting lactols were successively treated with acetic anhydride to promote the ring opening of the lactol, and with tosyl hydrazide to give the corresponding hydrazones 192, which were isolated as a single isomer in good to excellent overall yields from amine $\mathbf{1 9 0}$ without purification of synthetic intermediates (Scheme 25). 


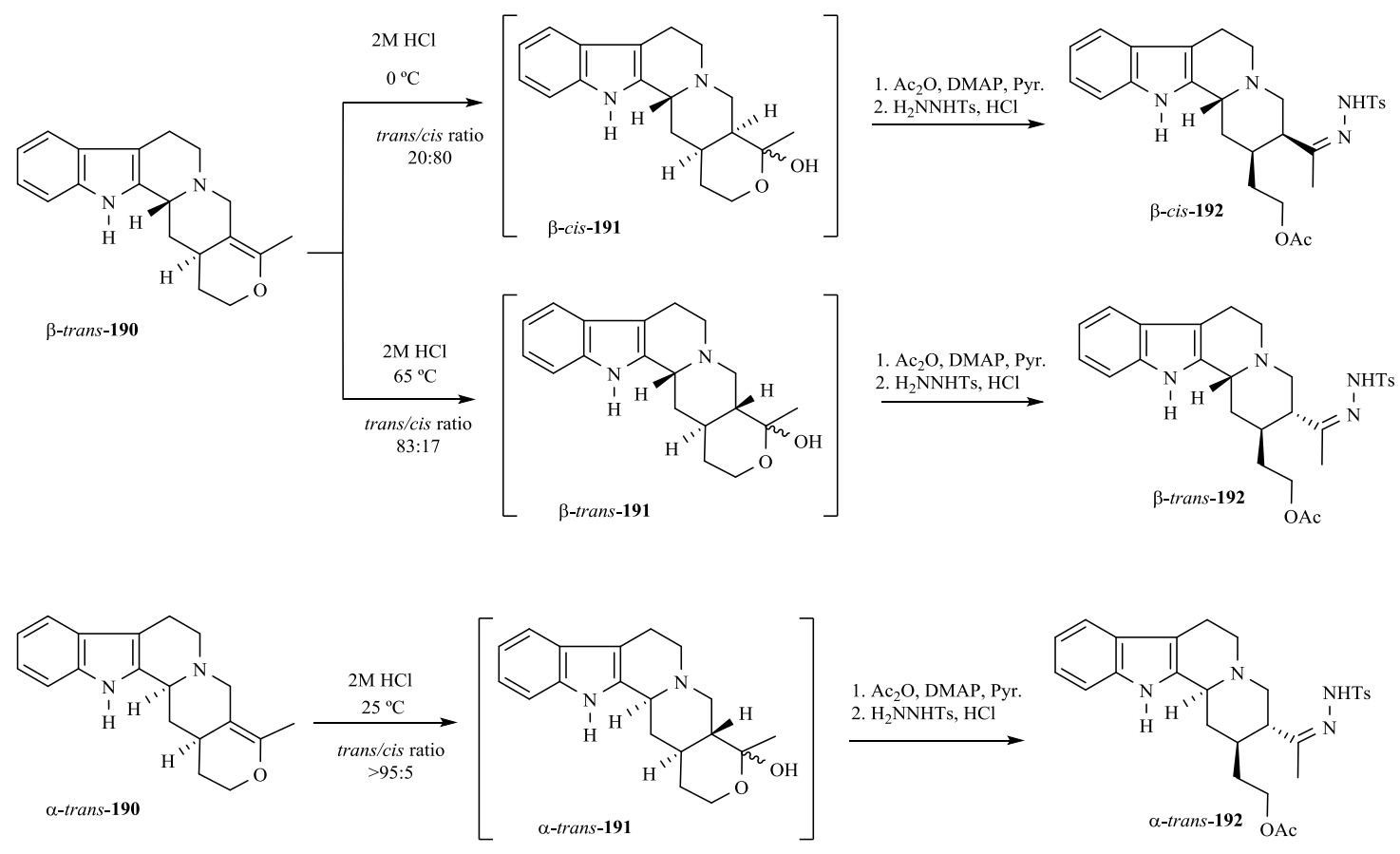

Scheme 25 - Preparation of the hydrazones 192.

Hydrazones $12 \mathrm{~b}-\alpha$-trans-192 and $12 \mathrm{~b}-\beta$-trans-192 were reduced with $\mathrm{NaBH}_{3} \mathrm{CN}$ in the presence of $\mathrm{HCl}$. Final addition of $\mathrm{KOH}$ aq. in methanol gave the natural product (-)dihydrocorynantheol (3) and (+)-hirsutinol (182). On the other hand, treatment of hydrazones $\alpha$ trans-192 and $\beta$-trans-192 with $n$-BuLi resulted in the elimination of the hydrazone and deacetylation providing access to (-)-corynantheol (183), whereas the oxidation of the hydroxyquinolizidines gave (-)-dihydrocorynantheal (184) and (-)-corynantheal (185) (Scheme 26). 

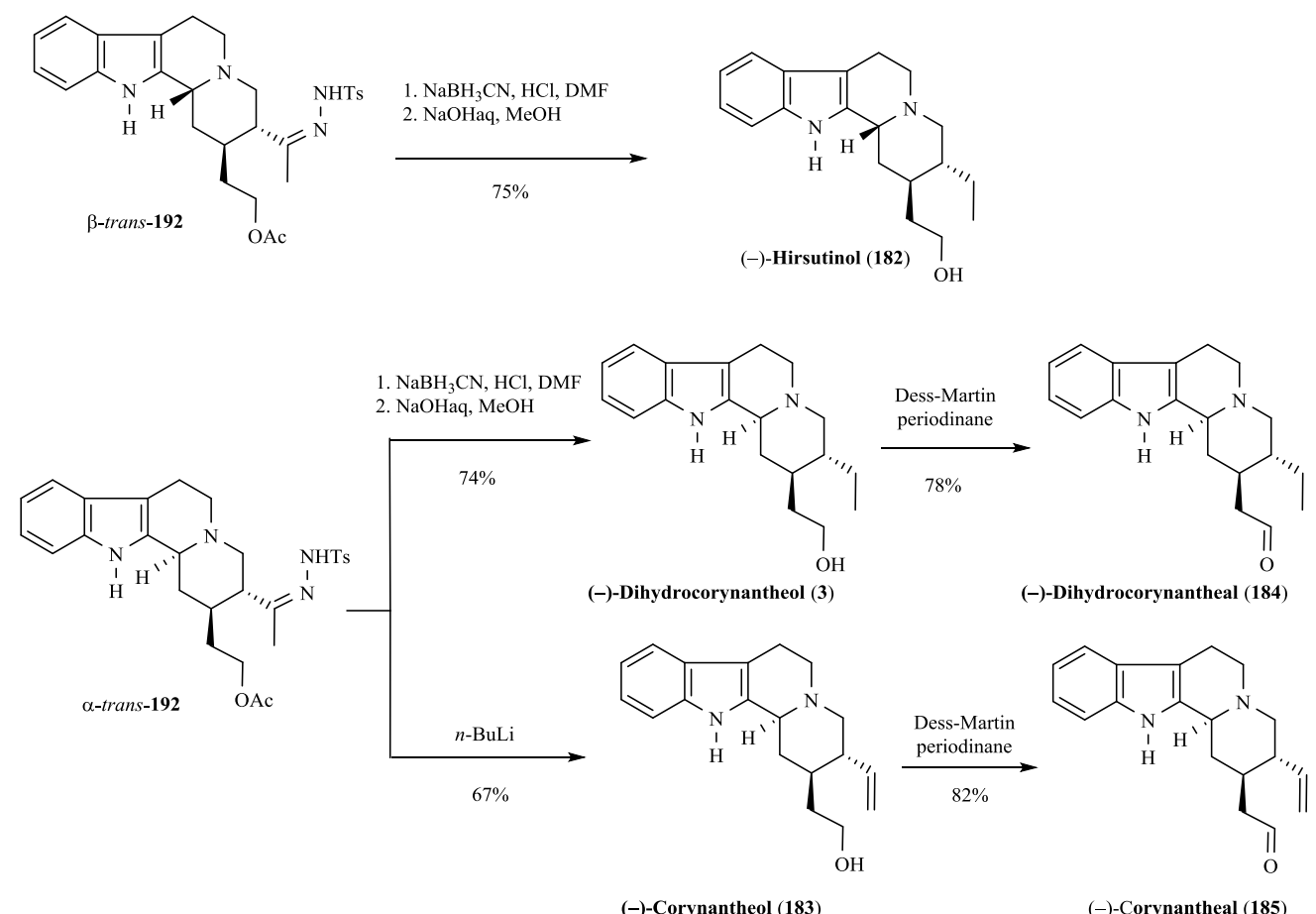

(-)-Corynantheal (185)

Scheme 26 - Preparation of Corynantheine alkaloids.

\subsubsection{Organocatalytic Enantioselective total synthesis of (-)-arboricine (28).}

A concise and scalable synthesis of (-)-arboricine (28) based on an asymmetric organocatalytic Pictet-Spengler reaction, followed by a $\mathrm{Pd}(0)$-catalyzed enolate cyclization, was described by Maarseveen and Hiemstra in 2009 (Scheme 27).[95]

The synthesis started with the know tryptamine 194,[96] which was prepared in one step by alkylation of tryptamine with Z-2-iodo-2-butene-1-ol mesylate (193) in $84 \%$ yield. PictetSpengler condensation of 194 with the dioxolane 195 using catalyst $(R)$-binol-PA (A), provided 196 in $92 \%$ yield and $78 \%$ ee. The acetal protecting group improves the ee of this reaction. The best ee was obtained using the sterically slightly more demanding catalyst $(R)$-H8-binol-PA $(\mathbf{B})$ that gave 196 in $86 \%$ yield and $89 \%$ ee. Treatment of 196 with $\mathrm{Boc}_{2} \mathrm{O}$ and DMAP followed by diluted $\mathrm{HCl}$ in acetone gave ketone $\mathbf{1 9 7}$ in $96 \%$ overall yield. The closure of the piperidine ring was accomplished by a $\mathrm{Pd}(0)$-catalyzed vinyl iodide-enolate coupling using the procedure published by Sole and Bonjoch, in which potassium phenoxide avoids the migration of the isolated exocyclic double bond[97] affording keto amine $\mathbf{1 9 8}$ as a single diastereoisomer. A 
single recrystallization from the EtOAc/PE mixture gave enantiometically pure 198. (-)arboricine was obtained in $81 \%$ yield after TFA-mediated removal of the Boc-protecting group of 198.

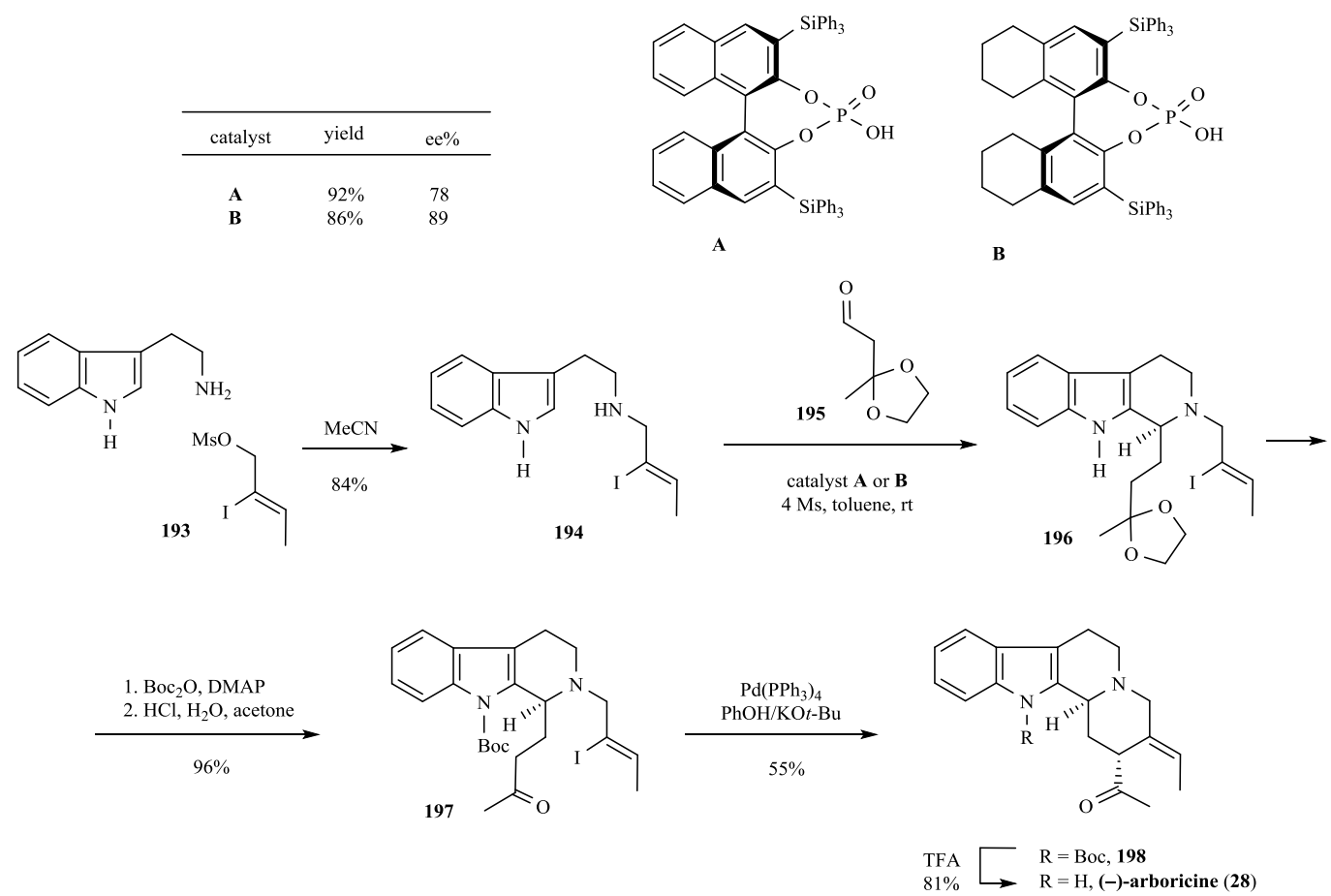

Scheme 27 - Organocatalytic enantioselective total synthesis of (-)-arboricine (28).

\subsubsection{Proline-catalyzed asymmetric addition reaction: Total synthesis of ent-}

\section{dihydrocorynantheol (3)}

In the context of Itoh's research on the synthesis of chiral 1 -substituted 1,2,3,4-tetrahydro- $\beta$ carboline derivatives, using the asymmetric addition of methyl ketones to the $\beta$-carboline system in the presence of $(S)$-proline,[98] this author envisaged that 3-ethyl-3-buten-2-one as a good substrate for the concise asymmetric synthesis of ent-dihydrocorynantheol (Scheme 28).[99]

The reaction of $N$-tosyl-3,4-dihydro- $\beta$-carboline (199) with 3-ethyl-3-buten-2-one (200)[100] in the presence of $(S)$-proline (201) led to the formation of the D ring of the target molecule with the correct configuration in a single step, affording the product $\mathbf{2 0 2}$ in a good yield of $85 \%$ with $99 \%$ ee. Since there were no intermediates observed in the reaction, it was not possible to 
conclude whether the reaction proceeded via Mannich-Michael reaction or a Diels-Alder-type addition. Alkene 203 was then quantitatively obtained in the ratio of $E / Z$ 1:20 from compound 202 by Wittig reaction. Subsequent treatment with Red-Al brought about the reductive elimination of the tosyl group and the reduction of the ester group to an alcohol. A final hydrogenation satisfactorily afforded ent-dihydrocorynantheol.

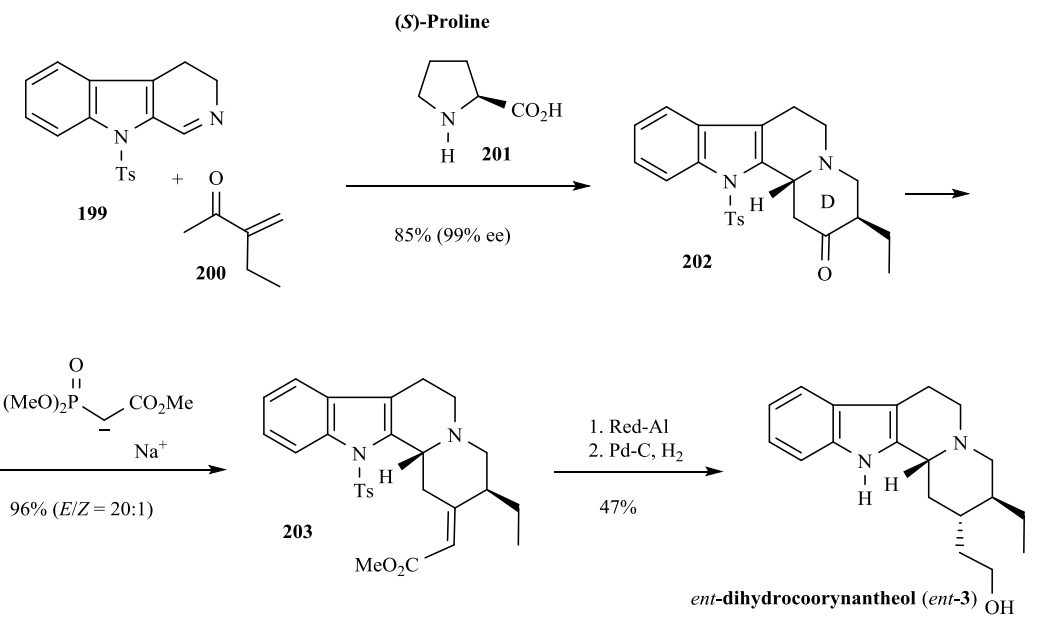

Scheme 28 - Total synthesis of ent-dihydrocorynantheol (ent-3).

\section{Final Remarks}

There has been much progress in the synthesis and biological evaluation of tetracyclic indole alkaloids containing an indolo[2,3-a]quinolizidine system. However, we can still find many reports describing the biological activity of extracts, instead of describing the activity of the pure indole alkaloids. Also, many reports do not describe correctly the stereochemistry of the indole alkaloids isolated and/or evaluated.

Herein, we have made a review about the potential pharmacological applications of indolo[2,3a]quinolizidines and derivatives (see Table 1), as well as about the most important asymmetric approaches developed for the construction of the indolo[2,3-a]quinolizine ring system. Although, to our knowledge, there are no alkaloids in the market or in clinical assays containing an indolo[2,3-a]quinolizidine system, compounds containing a tetracyclic indole[2,3-a]quinolizidine scaffold seem to be a potential starting point for the development of effective drugs. 


\begin{abstract}
Abbreviations
Ac, acetyl; Boc, tert-Butyloxycarbonyl; DHC, dihydrocorynantheol; Me, methyl; Nuclear factor-kB, NF-kB; OGlu, $O-\beta$-D-glucosyl; SIRT, sirtuin; DIBAL-H, diisobutylaluminium hydride; HWE, Horner-Wadsworth-Emmons; THF, tetrahydrofuran; DMAP, 4dimethylaminopyridine; PPTS, Pyridinium $p$-toluenesulfonate; TBSCl, tert-Butyldimethylsilyl chloride;
\end{abstract}

\title{
Acknowledgements
}

Fundação para a Ciência e a Tecnologia (FCT, Portugal) through iMed.ULisboa (UID/DTP/04138/2013), MINECO (Spain, project CTQ2012-35250), and AGAUR (Generalitat de Catalunya, Grant 2014SGR-0155) are gratefully acknowledged.

\section{References}

[1] Brown RT. Indoles. The Monoterpenoid Indole Alkaloids. In: Saxton JE, Ed. The Chemistry of Heterocyclic Compounds; Weissberger A, Taylor, EC Eds.;New York: Wiley 1983, Vol. 25, Part 4, Chapter 3.

[2] Szántay C, Blaskó G, Honty K, et al. The Alkaloids. In: Brossi A, Ed. London: Academic Press 1986, Vol. 27, Chapter 2.

[3] Lounasmaa M, Tolvanen A. Monoterpenoid Indole Alkaloids. In: Saxton JE, Ed. The Chemistry of Heterocyclic Compounds. Chichester: Wiley 1994, Vol.25, Part 4, Chapter 3.

[4] Johns SR, Lamberton JA, Occolowitz JL. 1,2,3,4,6,7-Hexahydro-12H-indolo[2,3a]quinolizine an alkaloid from Dracontomelum Mangiferum bl. (family anacardiaceae). Aust J Chem 1966; 19: 1951-1954. 
[5] Ito Y, Yano S, Watanabe K, et al. Structure activity relationship of yohimbine and its related analogs in blocking alpha-1 and alpha-2 adrenoceptors - a comparative-study of cardiovascular activities. Chem Pharm Bull 1990; 38: $1702-1706$.

[6] Chbani M, Pais M, Delauneux JM, et al. Brominated indole alkaloids from the marine tunicate Pseudodistoma arborescens. J Nat Prod 1993; 56: 99-104.

[7] Santos LS, Theoduloz C, Pilli RA, et al. Antiproliferative activity of arborescidine alkaloids and derivatives. Eur J Med Chem 2009; 44: 3810-3815.

[8] Chang P, Koh YK, Geh SL, et al. Cardiovascular effects in the rat of dihydrocorynantheine isolated from uncaria-callophylla. J Ethnopharmacol 1989; 25: 213-215.

[9] Masumiya $H$, Saitoh $T$, Tanaka $Y$, et al. Effects of hirsutine and dihydrocorynantheine om the action potentials of sino-atrial node, atrium and ventricle. Life Sci 1999; 65: 2333-2341.

[10] Staerk D, Lemmich E, Christensen J, et al. Leishmanicidal, antiplasmodial and cytotoxic activity of indole alkaloids from Corynanthe pachyceras. Planta Med 2000; 66: 531-536.

[11] Liz R, Pereira DF, Horst H, et al. Protected effect of Esenbeckia leiocarpa upon the inflammatory response induced by carrageenan in a murine air pouch model. Int Immunopharmacol 2011; 11: 1991-1999.

[12] Pozzatti P, dos Reis GO, Pereira DF, et al. Relationship of chemical structure and anti-inflammatory activity of dihydrocorynantheol and its analogues. Pharmacol Rep 2013; 65: 1263-1271. 
[13] Horie S, Yano S, Aimi N, et al. Effects of hirsutine, an antihypertensive indole alkaloid from uncaria-rhynchophylla, on intracellular calcium in rat thoracic aorta. Life Sci 1992; 50: 491-498.

[14] Wu LX, Gu XF, Zhu YC, et al. Protective effects of novel single compound, Hirsutine on hypoxic neonatal rat cardiomyocytes. Eur J Pharmacol 2011; 650: 290-297.

[15] Yuzurihara M, Ikarashi Y, Goto K, et al. Geissoschizine methyl ether, an indole alkaloid extracted from Uncariae Ramulus et Uncus, is a potent vasorelaxant of isolated rat aorta. Eur J Pharmacol 2002; 444: 183-189.

[16] Matsuo H, Okamoto R, Zaima K, et al. New vasorelaxant indole alkaloids, villocarines A-D from Uncaria villosa. Bioorg Med Chem 2011; 19: 4075-4079.

[17] Pozzatti P, dos Reis GO, Pereira DF, et al. Esenbeckia leiocarpa Engl. inhibits inflammation in a carrageenan-induced murine model of pleurisy. J Pharm Pharmacol 2011; 63: 1091-1102.

[18] Ueda T, Ugawa S, Ishida $Y$, et al. Geissoschizine methyl ether has thirdgeneration antipsychotic-like actions at the dopamine and serotonin receptors. Eur J Pharmacol 2011; 671: 79-86.

[19] Yang Z-d, Duan D-z, Du J, et al. Geissoschizine methyl ether, a corynantheantype indole alkaloid from Uncaria rhynchophylla as a potential acetylcholinesterase inhibitor. Nat Prod Res 2012; 26: 22-28.

[20] Cardoso CL, Silva DHS, Tomazela DM, et al. Turbinatine, a potential key intermediate in the biosynthesis of corynanthean-type indole alkaloids. J Nat Prod 2003; 66: 1017-1021. 
[21] Jung HY, Nam KN, Woo B-C, et al. Hirsutine, an indole alkaloid of Uncaria rhynchophylla, inhibits inflammation-mediated neurotoxicity and microglial activation. Mol Med Rep 2013; 7: 154-158.

[22] Qi W, Yue S-J, Sun J-H, et al. Alkaloids from the hook-bearing branch of Uncaria rhynchophylla and their neuroprotective effects against glutamate-induced HT22 cell death. J Asian Nat Prod Res 2014; 16: 876-883.

[23] Lou C, Takahashi K, Irimura T, et al. Identification of Hirsutine as an antimetastatic phytochemical by targeting NF-kappa B activation. Int J Oncol 2014; 45: 2085-2091.

[24] Lim K-H, Hiraku O, Komiyama K, et al. Biologically active indole alkaloids from Kopsia arborea. J Nat Prod 2007; 70: 1302-1307.

[25] Mukhopadhyay S, Handy GA, Funayama S, et al. Anticancer indole alkaloids of rhazya-stricta. J Nat Prod 1981; 44: 696-700.

[26] Soares PRO, de Oliveira PL, de Oliveira CMA, et al. In vitro antiproliferative effects of the indole alkaloid vallesiachotamine on human melanoma cells. Arch Pharm Res 2012; 35: 565-571.

[27] Passos CdS, Soldi TC, Abib RT, et al. Monoamine oxidase inhibition by monoterpene indole alkaloids and fractions obtained from Psychotria suterella and Psychotria laciniata. J Enzyme Inhib Med Chem 2013; 28: 611-618.

[28] Passos CS, Simoes-Pires CA, Nurisso A, et al. Indole alkaloids of Psychotria as multifunctional cholinesterases and monoamine oxidases inhibitors. Phytochem 2013; 86: 8-20.

[29] Sacconnay L, Ryckewaert L, Passos CdS, et al. Alkaloids from psychotria target sirtuins: in silico and in vitro interaction studies. Planta Med 2015; 81: 517-524. 
[30] Matsumoto K, Yamamoto LT, Watanabe K, et al. Inhibitory effect of mitragynine, an analgesic alkaloid from Thai herbal medicine, on neurogenic contraction of the vas deferens. Life Sci 2005; 78: 187-194.

[31] Kitajima M, Misawa K, Kogure $N$, et al. A new indole alkaloid, 7hydroxyspeciociliatine, from the fruits of Malaysian Mitragyna speciosa and its opioid agonistic activity. J Nat Med 2006; 60: 28-35.

[32] Matsumoto K, Horie S, Ishikawa H, et al. Antinociceptive effect of 7hydroxymitragynine in mice: Discovery of an orally active opioid analgesic from the Thai medicinal herb Mitragyna speciosa. Life Sci 2004; 74: 2143-2155.

[33] Horie S, Koyama F, Takayama H, et al. Indole alkaloids of a Thai medicinal herb, Mitragyna speciosa, that has opioid agonistic effect in guinea-pig ileum. Planta Med 2005; 71: 231-236.

[34] Matsumoto $\mathrm{K}$, Takayama $\mathrm{H}$, Ishikawa $\mathrm{H}$, et al. Partial agonistic effect of 9hydroxycorynantheidine on $\mu$-opioid receptor in the guinea-pig ileum. Life Sci 2006; 78: 2265-2271.

[35] Shamima AR, Fakurazi S, Hidayat MT, et al. Antinociceptive action of isolated mitragynine from mitragyna speciosa through activation of opioid receptor system. Int J Mol Sci 2012; 13: 11427-11442.

[36] Matsumoto K, Narita M, Muramatsu $N$, et al. Orally active opioid $\mu / \delta$ dual agonist MGM-16, a derivative of the indole alkaloid mitragynine, exhibits potent antiallodynic effect on neuropathic pain in mice. J Pharmacol Exp Ther 2014; 348: 383-392.

[37] Takayama H, Ishikawa H, Kurihara M, et al. Studies on the synthesis and opioid agonistic activities of mitragynine-related indole alkaloids: Discovery of opioid 
agonists structurally different from other opioid ligands. J Med Chem 2002; 45: 1949-1956.

[38] Wehner F, Noeren-Mueller A, Mueller O, et al. Indoloquinolizidine derivatives as novel and potent apoptosis inducers and cell-cycle blockers. Chembiochem 2008; 9: 401-405.

[39] Vendrell M, Soriano A, Casado V, et al. Indoloquinolizidine-peptide hybrids as multiple agonists for D-1 and D-2 dopamine receptors. Chemmedchem 2009; 4: $1514-1522$.

[40] Soriano A, Vendrell M, Gonzalez S, et al. A hybrid indoloquinolizidine peptide as allosteric modulator of dopamine D-1 receptors. J Pharmacol Exp Ther 2010; 332: $876-885$.

[41] Zhu K, Yang S-N, Ma F-F, et al. The novel analogue of hirsutine as an antihypertension and vasodilatary agent both in vitro and in vivo. PloS one 2015; 10: e0119477-e0119477.

[42] Ohba M, Ohashi T, Fujii T. Quinolizidines. XXIX. Preparation of (-)-dihydrocorynantheol. Heterocycles 1991; 32: 319-328.

[43] Fujii T, Ohba M, Shimohata K, et al. Quinolizidines. XXIII. An alternative synthetic route to benzo[a]quinolizidine-type alangium alkaloids from ethyl cincholoiponate. Heterocycles 1987; 26: 2949-2957.

[44] Deiters A, Chen K, Eary CT, et al. Biomimetic entry to the sarpagan family of indole alkaloids: Total synthesis of (+)-geissoschizine and (+)-Nmethylvellosimine. J Am Chem Soc 2003; 125: 4541-4550.

[45] Borch RF. A new method for reduction of secondary and tertiary amides. Tetrahedron Lett 1968: 61-65. 
[46] Bohlmann C, Bohlmann R, Rivera EG, et al. Reactions with indole-derivatives .53. enantioselective total synthesis of (+)-geissoschizine and (-)-geissoschizol. Liebigs Ann Chem 1985: 1752-1763.

[47] Deiters A, Pettersson M, Martin SF. General strategy for the syntheses of corynanthe, tacaman, and oxindole alkaloids. J Org Chem 2006; 71: 6547-6561.

[48] Besselievre R, Cosson JP, Das BC, et al. Structure and total synthesis of deplancheine, a novel indoloquinolizidine alkaloid. Tetrahedron Lett 1980; 21: 63-66.

[49] Sydorenko N, Zificsak CA, Gerasyuto Al, et al. Total syntheses of enantiomerically enriched $R-(+)$ - and $S$-(-)-deplancheine. Org Biomol Chem $2005 ; 3: 2140-2144$.

[50] Takasu K, Nishida N, Tomimura A, et al. Convenient synthesis of substituted piperidinones from alpha,beta-unsaturated amides: Formal synthesis of deplancheine, tacamonine, and paroxetine. J Org Chem 2005; 70: 3957-3962.

[51] Allin SM, Thomas $\mathrm{Cl}$, Doyle $\mathrm{K}$, et al. An asymmetric synthesis of both enantiomers of the indole alkaloid deplancheine. J Org Chem 2005; 70: 357359.

[52] Mondal P, Argade NP. Enantioselective Total Synthesis of Desbromoarborescidines A-C and the Formal Synthesis of (S)-Deplancheine. J Org Chem 2013; 78: 6802-6808.

[53] Martin SF, Chen KX, Eary CT. An enantioselective total synthesis of (+)geissoschizine. Org Lett 1999; 1: 79-81. 
[54] Amat M, Santos MMM, Bassas O, et al. Straightforward methodology for the enantioselective synthesis of benzo[a]- and indolo[2,3-a] quinolizidines. J Org Chem 2007; 72: 5193-5201.

[55] Pérez M, Arioli F, Rigacci G, et al. Stereocontrolled generation of benzo[a]- and indolo[2,3-a]quinolizidines from $\quad(S)$-Tryptophanol and $\quad(S)-(3,4-$ dimethoxyphenyl)alaninol-derived lactams. Eur J Org Chem 2011: 3858-3863.

[56] Amat M, Santos MMM, Gómez AM, et al. Enantioselective spirocyclizations from tryptophanol-derived oxazolopiperidone lactams. Org Lett 2007; 9: 29072910.

[57] Amat M, Gómez Esqué A, Escolano C, et al. Enantioselective formal synthesis of (+)-dihydrocorynantheine and (-)-dihydrocorynantheol. J Org Chem 2009; 74: $1205-1211$.

[58] Lounasmaa M, Hanhinen P. Studies on the biomimetic preparation of the sarpagan ring system. Attempts to apply the spontaneous "biogenetic-type cyclization" of van Tamelen to bond formation between C-5 and C-16 in the corynantheine series. Tetrahedron 1996; 52: 15225-15242.

[59] Lounasmaa M, Jokela R, Tirkkonen B, et al. Syntheses of ( \pm )-Z-geissoschizol, ( \pm )3-epi-Z-geissoschizol, ( \pm )-corynantheidol, ( \pm )-dihydrocorynantheol, ( \pm )-3-epidihydrocorynantheol and the corresponding corynan-17-oic acid methyl-esters. Heterocycles 1992; 34: 321-339.

[60] Ma J, Yin W, Zhou H, et al. Total synthesis of the opioid agonistic indole alkaloid mitragynine and the first total syntheses of 9-methoxygeissoschizol and 9methoxy- $N_{\mathrm{b}}$-methylgeissoschizol. Org Lett 2007; 9: 3491-3494. 
[61] Ley SV, Priour A. Total synthesis of the cyclic peptide argyrin B. Eur J Org Chem 2002: 3995-4004.

[62] Ley SV, Priour A, Heusser C. Total synthesis of the cyclic heptapeptide argyrin B: A new potent inhibitor of T-cell independent antibody formation. Org Lett 2002; 4: 711-714.

[63] Larock RC, Yum EK. Synthesis of indoles via palladium-catalyzed heteroannulation of internal alkynes. J Am Chem Soc 1991; 113: 6689-6690.

[64] Larock RC, Yum EK, Refvik MD. Synthesis of 2,3-disubstituted indoles via palladium-catalyzed annulation of internal alkynes. J Org Chem 1998; 63: 76527662.

[65] Liu XX, Deschamp JR, Cook JM. Regiospecific, enantiospecific total synthesis of the alkoxy-substituted indole bases, 16-epi- $N_{\mathrm{a}}$-methylgardneral, 11methoxyaffinisine, and 11-methoxymacroline as well as the indole alkaloids alstophylline and macralstonine. Org Lett 2002; 4: 3339-3342.

[66] Castle SL, Srikanth GSC. Catalytic asymmetric synthesis of the central tryptophan residue of celogentin C. Org Lett 2003; 5: 3611-3614.

[67] Zhou H, Liao XB, Cook JM. Regiospecific, enantiospecific total synthesis of the 12-alkoxy-substituted indole alkaloids, (+)-12-methoxy- $N_{\mathrm{a}}$-methylvellosimine, (+)-12-methoxyaffinisine, and (-)-fuchsiaefoline. Org Lett 2004; 6: 1187-1187.

[68] Yu JM, Wearing XYZ, Cook JM. Stereocontrolled total synthesis of (-)vincamajinine and (-)-11-methoxy-17-epivincamajine. J Am Chem Soc 2004; 126: 1358-1359.

[69] Schollkopf U, Groth U, Deng C. Enantioselective synthesis of $(R)$-amino acids using L-valine as chiral agent. Angew Chem Int Ed Engl 1981; 20: 798-799. 
[70] Ma CR, Liu XX, Li XY, et al. Efficient asymmetric synthesis of biologically important tryptophan analogues via a palladium-mediated heteroannulation reaction. J Org Chem 2001; 66: 4525-4542.

[71] Takayama H, Maeda M, Ohbayashi S, et al. The first total synthesis of (-)mitragynine, an analgesic indole alkaloid in Mitragyna speciosa. Tetrahedron Lett 1995; 36: 9337-9340.

[72] Yu S, Berner OM, Cook JM. General approach for the synthesis of indole alkaloids via the asymmetric Pictet-Spengler reaction: First enantiospecific total synthesis of (-)-corynantheidine as well as the enantiospecific total synthesis of (-)-corynantheidol, (-)-geissoschizol, and (+)-geissoschizine. J Am Chem Soc 2000; 122: 7827-7828.

[73] Nicolaou KC, Edmonds DJ, Bulger PG. Cascade reactions in total synthesis. Angew Chem Int Ed 2006; 45: 7134-7186.

[74] Tietze LF. Comprehensive chirality. Elsevier 2012.

[75] Pellissier H. Asymmetric domino reactions. Part A: Reactions based on the use of chiral auxiliaries. Tetrahedron 2006; 62: 1619-1665.

[76] Tietze LF, Beifuss U. Sequential transformations in organic-chemistry - A synthetic strategy with a future. Angew Chem Int Ed Engl 1993; 32: 131-163.

[77] Hua DH, Bharathi SN, Panangadan JAK, et al. Stereoselective additions of chiral $\alpha$-sulfinyl ketimine anions to ene esters - asymmetric syntheses of indolo 2,3- $a$ quinolizidine and yohimban alkaloids. J Org Chem 1991; 56: 6998-7007.

[78] Tietze LF, Zhou YF. Highly efficient, enantioselective total synthesis of the active anti-influenza A virus indole alkaloid hirsutine and related compounds by domino reactions. Angew Chem Int Ed 1999; 38: 2045-2047. 
[79] Tosaka A, Ito S, Miyazawa N, et al. Total synthesis of (-)-dihydrocorynantheol using bicyclo[3.2.1]octenone chiral building block. Heterocycles 2006; 70: 153159.

[80] Miyazawa N, Ogasawara K. A concise enantiocontrolled route to yohimbones using a bicyclo]3.2.1] octane chiral building block. Tetrahedron Lett 2002; 43: $4773-4776$.

[81] Tsunoda T, Suzuki M, Noyori R. A facile procedure for acetalization under aprotic conditions. Tetrahedron Lett 1980; 21: 1357-1358.

[82] Kobayashi T, Takeuchi K, Miwa J, et al. Efficient synthesis of 2,4,5-trisubstituted 2,5-chiral tetrahydropyridines using a one-pot asymmetric azaelectrocyclization protocol. Chem Commun 2009: 3363-3365.

[83] Li Y, Kobayashi T, Katsumura S. Synthesis of indole alkaloid (-)-corynantheidol and formal synthesis of (-)-corynantheidine via one-pot asymmetric azaelectrocyclization. Tetrahedron Lett 2009; 50: 4482-4484.

[84] Amat M, Hadida S, Pshenichnyi G, et al. Palladium(0)-catalyzed heteroarylation of 2- and 3-indolylzinc derivatives. An efficient general method for the preparation of (2-pyridyl)indoles and their application to indole alkaloid synthesis. J Org Chem 1997; 62: 3158-3175.

[85] English BJ, Williams RM. A divergent strategy for the synthesis of secologanin derived natural products. J Org Chem 2010; 75: 7869-7876.

[86] English BJ, Williams RM. Synthesis of ( \pm )-oleocanthal via a tandem intramolecular Michael cyclization-HWE olefination. Tetrahedron Lett 2009; 50: 2713-2715. 
[87] Dalko PI, Moisan L. In the golden age of organocatalysis. Angew Chem Int Ed 2004; 43: 5138-5175.

[88] Kumar P, Dwivedi N. Proline Catalyzed a-Aminoxylation Reaction in the Synthesis of Biologically Active Compounds. Acc Chem Res 2013; 46: 289-299.

[89] Gomez C, Betzer J-F, Voituriez A, et al. Phosphine Organocatalysis in the Synthesis of Natural Products and Bioactive Compounds. Chemcatchem 2013; 5: 1055-1065.

[90] Santos LS, Pilli RA, Rawal VH. Enantioselective total syntheses of (+)arborescidine A, (-)-arborescidine B, and (-)-arborescidine C. J Org Chem 2004; 69: 1283-1289.

[91] Schumacher RW, Davidson BS. Synthesis of didemnolines A-D, N9-substituted $\beta$-carboline alkaloids from the marine ascidian Didemnum sp. Tetrahedron 1999; 55: 935-942.

[92] Kobayashi S, Ishitani H. Catalytic enantioselective addition to imines. Chem Rev 1999; 99: 1069-1094.

[93] Yamakawa $M$, Ito $H$, Noyori R. The metal-ligand bifunctional catalysis: A theoretical study on the ruthenium(II)-catalyzed hydrogen transfer between alcohols and carbonyl compounds. J Am Chem Soc 2000; 122: 1466-1478.

[94] Zhang W, Bah J, Wohlfarth A, et al. A Stereodivergent Strategy for the Preparation of Corynantheine and Ipecac Alkaloids, Their Epimers, and Analogues: Efficient Total Synthesis of (-)-Dihydrocorynantheol, (-)Corynantheol, (-)-Protoemetinol, (-)-Corynantheal, (-)-Protoemetine, and Related Natural and Nonnatural Compounds. Chem Eur J 2011; 17: 1381413824. 
[95] Wanner MJ, Boots RNA, Eradus B, et al. Organocatalytic Enantioselective Total Synthesis of (-)-Arboricine. Org Lett 2009; 11: 2579-2581.

[96] Takayama $\mathrm{H}$, Watanabe $\mathrm{F}$, Kitajima $\mathrm{M}$, et al. A radical cyclization strategy for the concise total synthesis of $( \pm)$-geissoschizine. Tetrahedron Lett $1997 ; 38$ : 5307-5310.

[97] Sole D, Urbaneja X, Bonjoch J. Palladium-catalyzed intramolecular coupling of amino-tethered vinyl halides with ketones, esters, and nitriles using potassium phenoxide as the base. Adv Synth Catal 2004; 346: 1646-1650.

[98] Itoh T, Yokoya M, Miyauchi K, et al. Proline-catalyzed asymmetric addition reaction of 9-tosyl-3,4-dihydro- $\beta$-carboline with ketones. Org Lett 2003; 5: 4301-4304.

[99] Itoh T, Yokoya M, Miyauchi K, et al. Total synthesis of ent-dihydrocorynantheol by using a proline-catalyzed asymmetric addition reaction. Org Lett 2006; 8: $1533-1535$.

[100] Faulkner DJ, Petersen MR. Application of claisen rearrangement to synthesis of trans trisubstituted olefinic bonds - synthesis of squalene and insect juvenilehormone. J Am Chem Soc 1973; 95: 553-563. 